Review

\title{
Cantilever torque magnetometry on coordination compounds: from theory to experiments
}

\author{
Mauro Perfetti \\ Department of Chemistry, University of Copenhagen, Universitetparken 5, 2100 Copenhagen, Denmark
}

\section{A R T I C L E I N F O}

\section{Article history:}

Received 19 May 2017

Accepted 15 August 2017

\section{Keywords:}

Cantilever torque magnetometry

Magnetic anisotropy

Lanthanides

Transition metals

Thin layers

Single crystals

\begin{abstract}
A B S T R A C T
Cantilever Torque Magnetometry (CTM) is one of the leading techniques to deeply understand magnetic anisotropy of coordination compounds. The knowledge of magnetic anisotropy is a mandatory requirement before proceeding with any future application related to the magnetic properties of coordination compounds, such as quantum computation or information storage. This review enlightens that CTM offers a unique combination of accuracy and precision to disentangle noncollinear contributions inside Single Crystals as well as the sensitivity to detect molecular order of thin films. CTM can also detect quantum phenomena such as magnetization steps and molecular hysteresis curves. Moreover, it can also provide the energy levels splitting and wavefunctions composition, especially if coupled with microwave radiation.
\end{abstract}

(c) 2017 Elsevier B.V. All rights reserved.

\section{Contents}

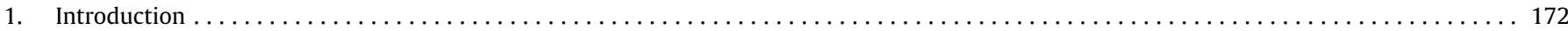

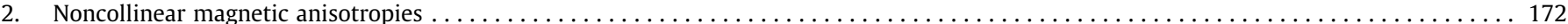

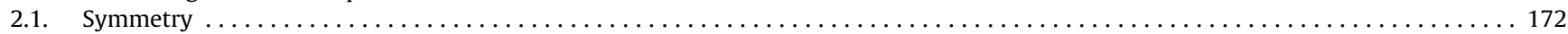

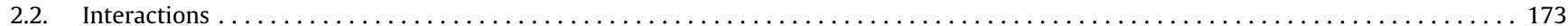

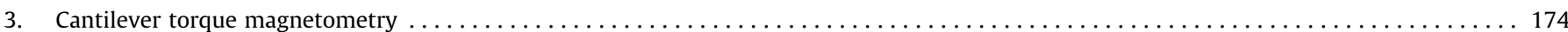

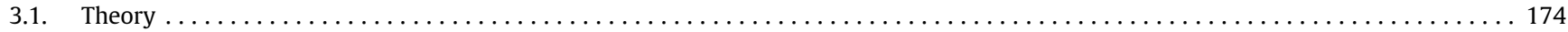

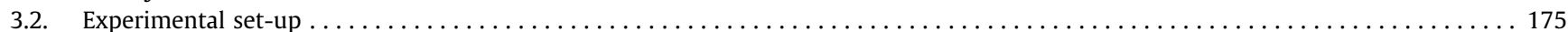

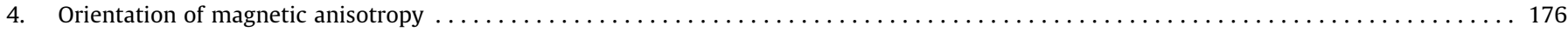

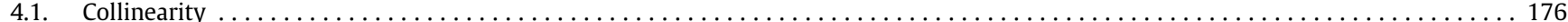

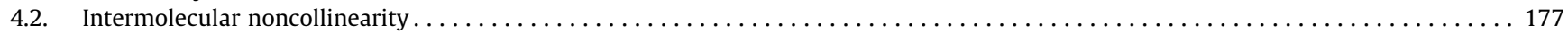

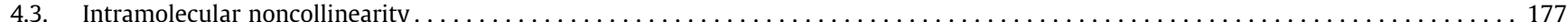

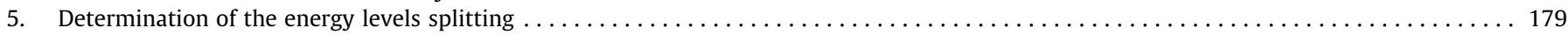

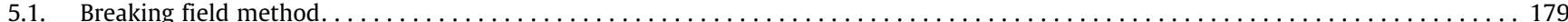

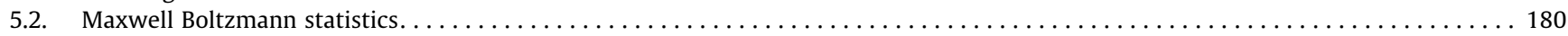

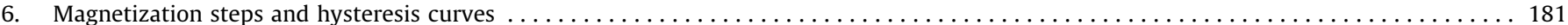

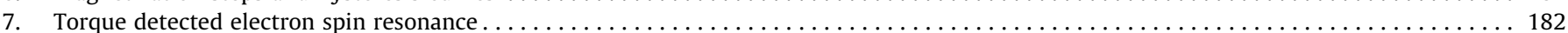

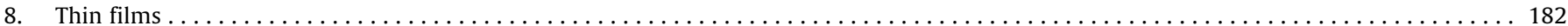

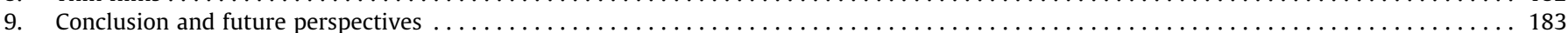

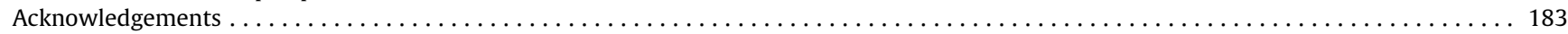

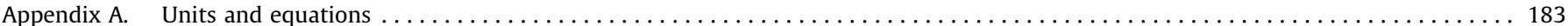

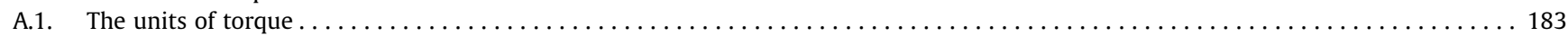

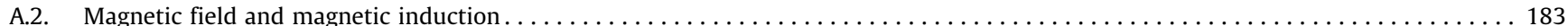

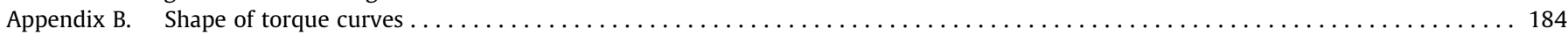

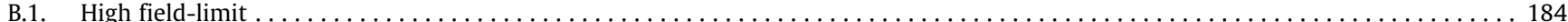

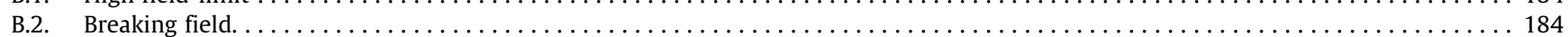

E-mail address: mauro.perfetti@chem.ku.dk 


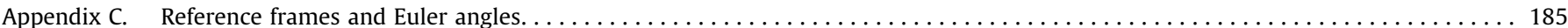

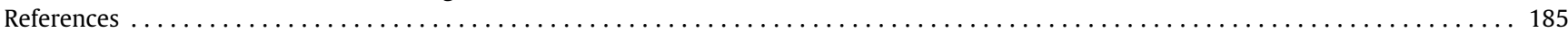

\section{Introduction}

Molecular magnetism is an interdisciplinary research area that embraces both chemistry and physics. The aim of the field is to understand, optimize and exploit the magnetic properties of molecular compounds containing unpaired electrons either delocalized over a portion of an organic molecule (radicals) or, more often, belonging to paramagnetic metals in coordination complexes. The intriguing future applications related to these molecules include information storage (e.g. Single Molecule Magnets, SMMs) [1], quantum calculations (e.g. Spin Quantum Bits) [2-5] and molecular quantum spintronics [6-9].

Using single molecules instead of extended magnetic networks (e.g. magnetic oxides or alloys) enables to chemically engineer the structure to obtain or tune a target characteristic. A property that must be known for all the aforementioned applications is magnetic anisotropy, defined as the directional dependence of the magnetic properties. From its definition, it is evident that the key to tailor the magnetic anisotropy of coordination complexes is to establish reliable magneto-structural correlations. The detailed explanation of the origin of magnetic anisotropy at the molecular level is beyond the aim of this review and can be found in several books $[10,11]$, however we must mention that the simplest building blocks that can be varied to tune magnetic anisotropy are the chemical nature and number of metal ions as well as the geometry, chemical composition and number of the coordinating ligands. A large fan of very different anisotropic coordination complexes has been synthesized so far [12-18]. Among them, two categories are particularly relevant in the context of this review: polynuclear transition metal-based clusters [16] and mononuclear lanthanide complexes [12].

Since magnetic anisotropy is intrinsically connected to a spatial dependence, its experimental study is usually performed using angular-resolved measurements on ordered systems (e.g. single crystals or oriented films). Among the several techniques that can extract information about the orientation and magnitude of magnetic anisotropy, the most known is Single Crystal Magnetometry (SCM), usually performed in a Superconducting Quantum Interference Device (SQUID) magnetometer [19-22]. Despite a quite good sensitivity, the technique has the major drawback to be mainly limited to systems in which the anisotropies are collinear (see also Section 2.1). Particularly sensitive are also spectroscopic techniques such as Electron Paramagnetic Resonance (EPR) [23-27] and Nuclear Magnetic Resonance (NMR) [28-34]. However, EPR is usually done only at very low temperatures and NMR often in solution. Polarized Neutron Diffraction (PND) for single crystal studies [35-37] and X-ray Natural Linear Dichroism (XNLD) for films [38-41] are powerful alternatives. The first one requires large crystals (typically hundreds of $\mathrm{mm}^{3}$ [36]), but can be applied both in the high field and in the low field regime and can provide access to the magnetization density of each individual crystallographic site. XNLD is mainly limited by the depth penetration of the employed radiation. The major drawback of both techniques is the necessity of large-scale facilities to generate neutrons and X-ray beams.

Cantilever torque magnetometry (CTM) constitutes an excellent alternative. Indeed, it requires an inexpensive experimental set-up (Section 3.2) and is extremely sensitive to magnetic anisotropy, being also able to disentangle noncollinear contributions (Section 4). This peculiarity allows to study crystals belonging to all the seven crystal systems, thus surpassing the major limit of SCM. The main disadvantage is that CTM allows to find a number of solutions equal to the number of noncollinear contributions to magnetic anisotropy, thus needing theoretical calculations or other experimental evidences to solve the ambiguity. Another extremely useful advantage of the technique is the possibility to investigate all kind of magnetic ions disregarding the parity of the spin (e.g. integer or half-integer), conversely from traditional low frequency X- or Q-band EPR. Concerning the ideal systems to investigate, the technique can be considered partially complementary to EPR, indeed CTM can be exploited at best with highly anisotropic samples (e.g. $\mathrm{Co}^{2+}$ or $\mathrm{Dy}^{3+}$ complexes) while EPR is more sensitive to small anisotropies (e.g. $\mathrm{Mn}^{2+}$ or $\mathrm{Gd}^{3+}$ complexes). Moreover, CTM can be used to investigate light crystals (few $\mu \mathrm{g}$ ) of both lanthanide and transition metal complexes up to room temperature, thus being more generally applicable than EPR. This provides information on all the energy levels that become significantly populated in a broad temperature range, giving access to the energy levels structure (Section 5). Furthermore, CTM allows to investigate systems that exhibit molecular hysteresis (Section 6). The technique has been coupled with microwave radiation (in which case is known as Torque Detected Electron Spin Resonance) to gain sensitivity towards quantum phenomena such as levels crossings (Section 7). Very recently, CTM was successfully used to study molecular order of thin films (Section 8).

The range of chemical structures of the coordination compounds studied via CTM is extremely broad. Since many of those are composed of several magnetic centres and/or form crystals that contain more than one noncollinear contribution to magnetic anisotropy, before proceeding with the discussion of theory and experiments, it is useful to briefly describe some guidelines to identify the origin of noncollinearity.

\section{Noncollinear magnetic anisotropies}

The complexity of a system increases with the number of noncollinear anisotropies that it contains because each of them exhibits in the general case a different magnetic response to an applied field. The sum of the anisotropies belonging to the magnetic centres of a molecule defines the molecular anisotropy, while the sum of the molecular anisotropies packed in a periodic way constitutes the crystal anisotropy. The number of noncollinear anisotropies inside a crystal depends on the chemical structure, on the crystallographic packing and on the presence of interactions between magnetic centres. Hereafter we discuss separately the relation that connects magnetic noncollinearity with symmetry (Section 2.1) and interactions (Section 2.2).

\subsection{Symmetry}

For mononuclear complexes, noncollinearity can arise due to the presence of crystallographically-inequivalent molecules and/ or to the presence of symmetry elements in the unit cell. In the former case, the unit cell contains several molecules not related by symmetry elements, thus noncollinearity should trivially arise. Note that this category includes both crystals formed by molecules with identical (see e.g. Ref. [42]) or different chemical structure (e.g. ionic pairs composed of magnetic anion and cation $[43,44]$ ). If instead all the molecules are crystallographically-equivalent, collinearity is strictly granted only when the molecule has the 
a)

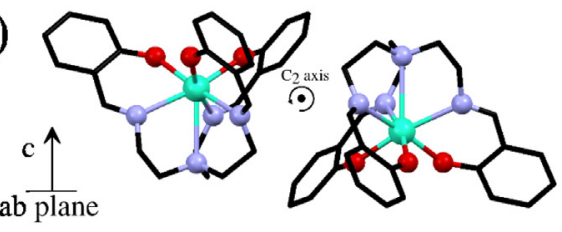

b)

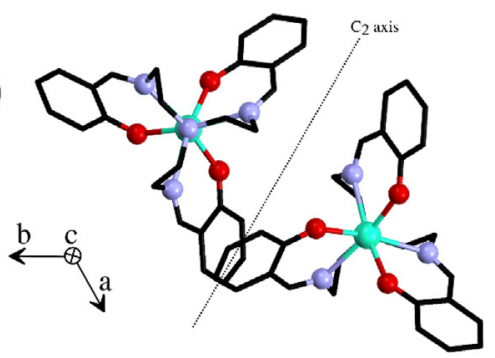

Fig. 1. DyTRENSAL structure seen from the $a b$ plane (a) and from the $c$ axis (b). Colour code: Dy-green, C-black, N-blue, O-red.

same point-group symmetry as the crystal structure, excluding the inversion. ${ }^{1}$ In this circumstance, the orientation of magnetic anisotropy can be obtained, without ambiguity, with all the experimental techniques mentioned in the Introduction. An obvious consequence is that highly symmetric crystal systems (e.g. cubic) are more prone to contain noncollinear anisotropies compared to low symmetry ones. In these cases, all the single crystal techniques can provide information about the crystal anisotropy but only few of them (mainly PND, EPR and Torque Magnetometry) can disentangle the single molecular contributions.

A powerful way to reduce the number of magnetically inequivalent entities to be included in the calculation or fit of single crystal data, is to approximate the molecular symmetry to the highest compatible with the ligands distance and geometry. Although evidences that inaccurate symmetry assumptions can lead to completely wrong results have been reported [19,20], a good symmetry approximation can drastically reduce the number of independent fitting parameters, allowing the analysis of otherwise complicated systems. In Fig. 1 we reported the structure of DyTRENSAL $\left(\mathrm{H}_{3}\right.$ TRENSAL $=2,2^{\prime}, 2^{\prime \prime}$-tris(salicylideneimino)triethyla mine) that crystallizes in the trigonal $P-3 c 1$ space group. The presence of an inversion centre reduces the number of noncollinear molecules in the unit cell from 4 to 2 (the ones depicted in Fig. 1). The symmetry operation that connects these two molecules is a $C_{2}$ axis lying in the $a b$ crystallographic plane. Since this symmetry operation does not belong to the point group of the molecule, the anisotropies of the two molecules reported in Fig. 1 are in principle noncollinear (the only symmetry restriction is that both $z$ molecular axes lie along $c$ ). However, considering the $a b$ plane magnetically isotropic (this assumption must be justified by experimental measurements, see also Section 4.1) it is possible to safely consider the anisotropies collinear and reduce the problem to the trivial determination of a single anisotropy.

The case of polynuclear clusters is more complicated. For these molecules, all the considerations related to crystallographic symmetry elements enlightened in the previous paragraph are still valid, however the presence of different sites in the molecule might produce more complicated responses. When the paramagnetic centres do not share the same chemical nature, the ligand field around each metal is different, the sites are not related by symmetry elements and no other experimental/theoretical indication is available, the system cannot be further simplified.

If instead the magnetic centres are related by one or more symmetry elements, the system can be simplified using pure symmetry arguments. An example is the $\mathrm{Fe}_{3} \mathrm{La}$ complex, reported in Fig. 2. This compound is a variation of the $\mathrm{Fe}_{4}$ unit (in which a central $\mathrm{Fe}(\mathrm{III})$ ion is surrounded by three $\mathrm{Fe}(\mathrm{III})$ ions arranged in a triangular fashion [46]). In $\mathrm{Fe}_{3} \mathrm{La}$, the central ion is substituted with a La (III) diamagnetic ion that has the effect of reducing the magnetic

\footnotetext{
${ }^{1}$ Magnetic anisotropy is even with respect to the inversion of the coordinate system.
}

interactions between the peripheral $\mathrm{Fe}(\mathrm{III})$ ions. Since in this molecule a $C_{3}$ axis passes through the central metal, the molecular anisotropy should have a principal axis perpendicular to the plane defined by the metals. The Fe(III) site symmetry is a distorted octahedron, thus the only restriction on the single ion anisotropies is imposed by the three $\mathrm{C}_{2}$ axes parallel to the Fe-La bonds (that must be principal anisotropy axes for all the sites). However, once the reference frame of the first anisotropy is defined, the other two can be simply obtained by a proper rotation of the first one (see Appendix $C$ for further information about reference frame rotations).

\subsection{Interactions}

If the paramagnetic centres interact, noncollinearity can be also imposed by interactions. Considering for simplicity a system composed of two interacting spins, we can write the exchange interaction term in the Hamiltonian as $[47,48]$ :

$\mathcal{H}_{\text {exc }}=\widehat{\mathbf{S}}_{1} \cdot \boldsymbol{J}_{\text {exc }} \cdot \widehat{\mathbf{S}}_{2}=-J_{12} \widehat{\mathbf{S}}_{1} \cdot \widehat{\mathbf{S}}_{2}+\widehat{\mathbf{S}}_{1} \cdot \boldsymbol{D}_{12} \cdot \widehat{\mathbf{S}}_{2}+\boldsymbol{d}_{12} \widehat{\mathbf{S}}_{1} \times \widehat{\mathbf{S}}_{2}$

The matrix $\boldsymbol{J}_{\text {exc }}$ that describes the interaction is not subjected to any restriction, thus is often useful to decompose the expression in three terms: a scalar one, a tensorial one and a vectorial one. The scalar (Heisenberg) contribution is isotropic (it does not contain directional features). It tends to align the spin parallel $\left(J_{12}>0\right)$ or antiparallel $\left(J_{12}<0\right)$ and is generally the biggest in magnitude. The tensorial (anisotropic) contribution is responsible for the alignment of the spins along a certain direction, determined by the matrix $\boldsymbol{D}_{12}$. The vectorial (antisymmetric or Dzyaloshinskii-Moriya) term contains a vector product between the spins, thus minimizes the energy

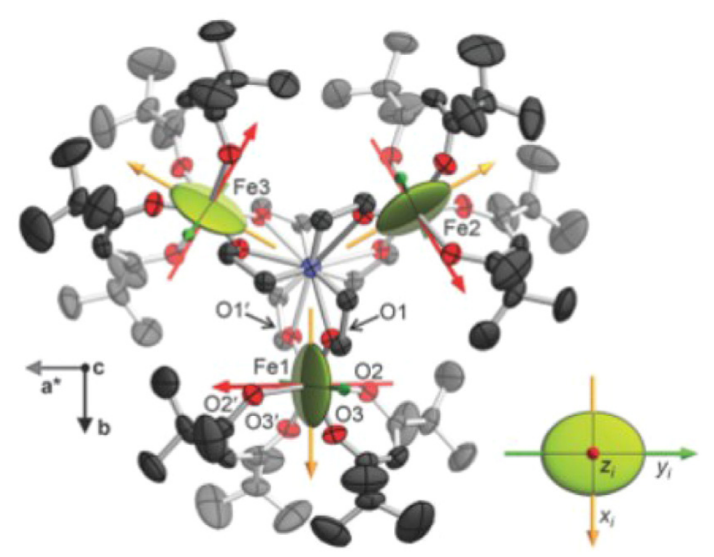

Fig. 2. Chemical and magnetic structure of the $\mathrm{Fe}_{3} \mathrm{La}$ molecule viewed along the threefold molecular axis (the $c$ crystallographic axis). The Fe atoms are replaced by single site susceptibility tensors drawn at an arbitrary scale. Arrows colour code: red-hard, yellow-intermediate, green-easy axis. Atoms colour code: C-black, O-red, La-blue, H were omitted for clarity. Image from Ref. [45]. 
when the spins are perpendicular to each other [49-51]. The resulting tilting angle between the spins is proportional to the ratio $\boldsymbol{d}_{12} /$ $\boldsymbol{J}_{\text {exc }}$ [48]. The Dzyaloshinskii-Moriya term can play a crucial role in defining noncollinearity as described for a ferric wheel [52] in Section 6 .

If the Heisenberg contribution to the magnetic coupling is the leading term in Eq. (1) and the temperature is sufficiently low (only the ground state is populated), the molecule can be safely considered as a unique magnetic entity with a well-defined value of the total spin $S$ (Giant Spin Approximation). In certain crystallographic symmetries, this approximation can be extremely useful: relevant examples for this review are the $\mathrm{Mn}_{12}$ clusters [53-55], the $\mathrm{Fe}_{4}$ family of SMM [46] and the transition metals-based metallic wheels [56,57] and grids [58].

Another source of noncollinearity is frustration, for example the geometrical impossibility to accommodate all the antiferromagnetic interactions at the same time [59-61]. Although frustration was firstly observed in extended networks, such as spin glasses [62-64], this phenomenon was demonstrated to be a crucial factor also in a number of molecular systems, for example in oddmembered rings [65] and triangular structures [66].

Using the considerations reported in Section 2, it is possible to identify the number and nature of noncollinear anisotropies to be expected in a target compound. This analysis is not simply useful, but instead mandatory, to meaningfully interpret the outcome of CTM measurements described in the next section.

\section{Cantilever torque magnetometry}

\subsection{Theory}

The torque, or moment of force, is the tendency of a force to transmit a rotation along a certain axis to an object. We experience torque very often in everyday life, for example whenever we open a door or we leaf through a book. The torque, often indicated as $\tau$, is proportional to the modulus of the applied force as well as to the modulus of the position vector (e.g. the vector that defines the physical quantity on which the force acts with respect to a chosen reference frame). Since the force tends to align the position vector parallel to itself, the mathematical expression that defines the magnetic torque is a simple vector product.

The magnetic analogous of classical torque is called magnetic torque. It defines the tendency of a magnetic dipole moment $\left(\mathbf{m}_{i}\right.$, belonging to the $i$-th atom) to align along the direction of the magnetic induction (B) locally acting on it

$\tau_{i}=\mathbf{m}_{i} \times \mathbf{B}=m_{i} B \sin \varphi_{i}$

where $\varphi_{i}$ is the angle between $\mathbf{m}_{i}$ and $\mathbf{B}$. For further details about the derivation of this formula and the approximations already introduced, the reader should refer to Appendix A. In samples where more than one magnetic moment is present, Eq. (2) can be simply extended summing over all the $N$ magnetic moments as follows:

$\tau=\sum_{i=1}^{N}\left(\mathbf{m}_{i} \times \mathbf{B}\right)=\mathbf{M} \times \mathbf{B}=M B \sin \varphi$

where $\mathbf{M}$ is the magnetization. Since Eq. (3) contains a vector product, $\tau$ is orthogonal to both $\mathbf{M}$ and $\mathbf{B}$ vectors (see Fig. 3 ).

The mathematical expression of $\tau$ in Eq. (3) strongly resembles the free energy $\left(E_{\text {free }}\right)$ of a dipole moment in a uniform magnetic field, where the angular dependence is sinusoidal instead of cosinusoidal. Moreover, the units of $\tau$ (Newton meter) are the same as an energy (see Appendix A for details about the units). It is thus possible to derive the expression of torque also from the free energy

$\tau_{\alpha}=-\left(\frac{\partial E_{f r e e}}{\partial \phi_{\alpha}}\right)_{B}$ where $\phi_{\alpha}$ is the angle that describes the rotation along the generic $\alpha$ axis.

Since this review is focused on the torque exerted by coordination compounds, we will now derive the equation that defines the magnetic torque of an anisotropic paramagnetic molecule inside a homogeneous magnetic field. To do so, it is useful to define the magnetic susceptibility tensor

$\chi_{i j}=\frac{\partial M_{j}}{\partial H_{i}}$

where $i, j=X, Y, Z$. For fields low enough $\left(k_{\mathrm{B}} T>g \mu_{\mathrm{B}} B\right) M$ increases linearly with $H$, thus $\chi$ becomes a simple ratio.

To calculate an analytical expression for the torque, we can start with the simplest example. Referring to Fig. 3, we can define a plane (say $X Z$ ) that contains both $\mathbf{M}$ and $\mathbf{B}$, so it holds

$M_{\alpha}=\chi_{\alpha \alpha} H_{\alpha}+\chi_{\beta \alpha} H_{\beta}$

where $\alpha=Z$ and $\beta=X$ and viceversa. In this arrangement, the magnetic torque vector is directed along the $Y$ axis. Substituting Eq. (6) into Eq. (3), we get

$$
\begin{aligned}
\tau_{Y} & =M_{Z} B_{X}-M_{X} B_{Z}=\frac{1}{\mu_{0}}\left[\left(\chi_{Z Z}-\chi_{X X}\right) B_{X} B_{Z}+\left(B_{X}^{2}-B_{Z}^{2}\right) \chi_{X Z}\right]= \\
& =\frac{1}{\mu_{0}}\left[B^{2}\left(\chi_{Z Z}-\chi_{X X}\right) \cos (\varepsilon) \sin (\varepsilon)+B^{2} \chi_{X Z}\left(2 \sin ^{2}(\varepsilon)-1\right)\right]
\end{aligned}
$$

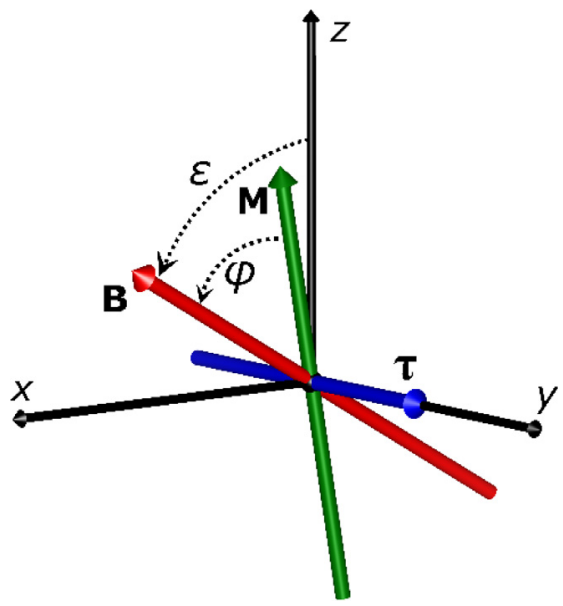

Fig. 3. Example of the orientation of $\mathbf{M}, \mathbf{B}$ and $\tau$ in an orthogonal reference frame $X Y Z$.

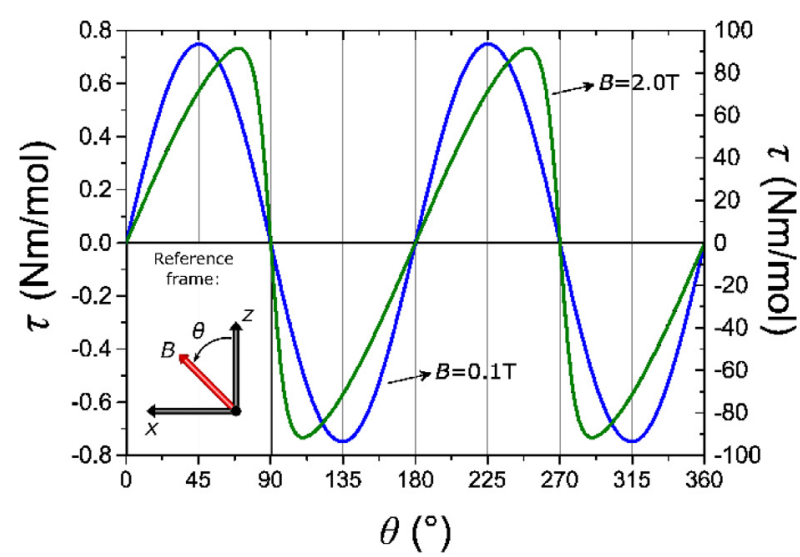

Fig. 4. Simulation of the magnetic torque exerted by a mole of $\mathrm{TbPc}_{2}$ molecules (parameters from Ref. [67]) at $\mathrm{T}=2 \mathrm{~K}$. The left scale refers to $\mathrm{B}=0.1 \mathrm{~T}$ (blue curve) while the right scale to $\mathrm{B}=2 \mathrm{~T}$ (green curve). The used reference frame system is reported in the inset. 

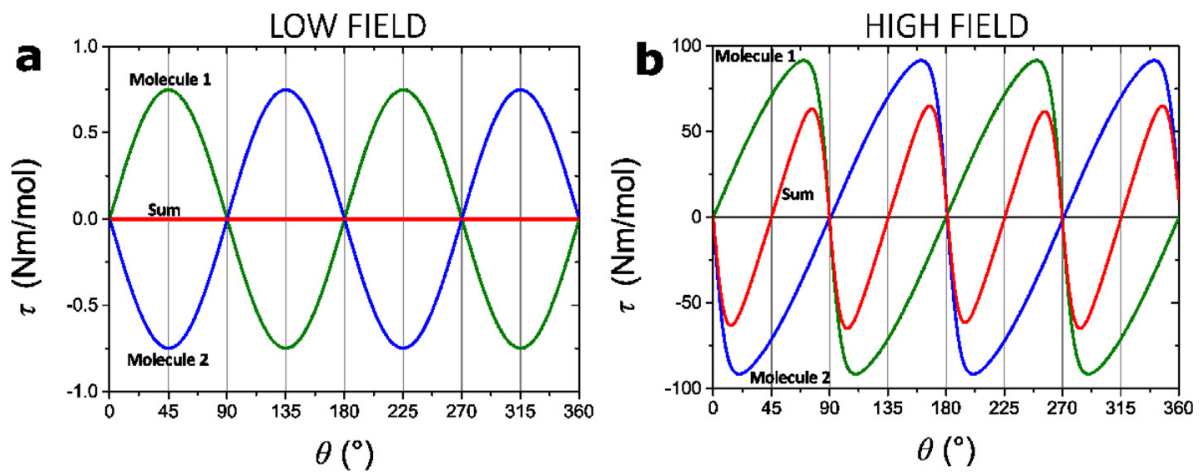

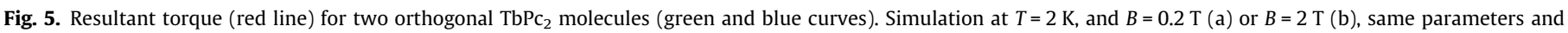
reference frame of Fig. 4.

where we conveniently defined $\varepsilon$ as the angle between the $Z$ axis and $\mathbf{B}$ (see Fig. 3). We can further simplify the picture in an axial system if we rotate the reference frame to let the $X$ and $Z$ directions coincide with the principal magnetic directions in the plane of rotation. In that case, the term dependent on $\chi_{X z}$ is identically zero, and we can finally write $\tau_{Y}$ as follows:

$\tau_{Y}=\frac{B^{2}}{\mu_{0}}\left(\chi_{Z Z}-\chi_{X X}\right) \cos (\varepsilon) \sin (\varepsilon)$

Eq. (8) contains several information about the anisotropy of the studied compound and thus deserves some comments. In the low field regime, torque is proportional to the anisotropic character of the magnetic susceptibility $\left(\chi_{z z}-\chi_{x x}\right)$. For this reason, Torque Magnetometry is particularly powerful if applied to highly anisotropic samples (e.g. lanthanides complexes). Moreover, for fields small enough, the signal is proportional to $B^{2}$, allowing to detect small signals even at moderate magnetic fields. From Eq. (8) it is also clear that the torque of an anisotropic paramagnet in the low field regime does not change sign every $k \pi(k=0,1,2 \ldots n$, integer) as expected for a conventional vector product, but instead it goes to zero every $k \pi / 2$. Zero-torque points occur both when $\mathbf{B}$ is parallel and when it is perpendicular to the easy axis, ${ }^{2}$ making Torque Magnetometry an ideal technique to detect the orientation of the principal axis of the magnetic anisotropy.

The blue line reported in Fig. 4 illustrates a particularly simple case: the magnetic torque exerted by a mole of a uniaxial system when the field is rotated in the $x z$ molecular magnetic reference frame. ${ }^{3}$ As sample molecule we chose $\left[\mathrm{TbPc}_{2}\right]^{-}(\mathrm{Pc}=$ dianion of phthalocyanine) due to its extensively characterized magnetic behaviour [67]. Following the reference frame depicted in Fig. 4, at $\theta=0^{\circ}$ the field is parallel to the easy axis of the molecule, thus the magnetic torque is zero ( $\mathbf{B}$ and $\mathbf{M}$ vectors are parallel). This point is commonly called "easy zero". For $0^{\circ}<\theta<90^{\circ}$ the sample tends to rotate $\mathbf{M}$ anticlockwise towards $\mathbf{B}$ causing a positive torque (maximum is reached at $\theta=45^{\circ}$ ). At $\theta=90^{\circ}$ the vector $\mathbf{B}$ is contained in the hard plane (perpendicular to the easy axis): since there are no components of $\mathbf{B}$ along the easy axis, the molecule is (poorly) magnetized again in the same direction of $\mathbf{B}$ and $\tau$ is again 0 ("hard zero"). An equivalent explanation of the zero-torque points can be formulated starting from Eq. (4): when B is applied along a free energy extreme torque must vanish. For both second order anisotropies and collinear axial systems the free energy extremes are orthogonal, thus a $90^{\circ}$

\footnotetext{
2 The term "easy" identifies the direction(s) along which a magnetic field produces the highest possible value of the magnetization while the opposite is true for the "hard" direction(s).

3 The magnetic reference frame $x y z$ is defined as the one in which the susceptibility tensor is diagonal, thus for the rotation showed in Fig. 4, the angle $\theta$ coincides with the angle $\varepsilon$ in Eq. (8)
}

periodicity of the zero-torque points must be always observed. Conversely, the inclusion of higher order terms in the description of magnetic anisotropy might remove this periodicity. ${ }^{4}$ An example is the low temperature magnetic torque of the DyTRENSAL complex, described in Section 4.1.

If the magnetic field is high enough and/or the thermal energy is sufficiently low (high field limit, $k_{\mathrm{B}} T<<g \mu_{\mathrm{B}} B$ ), the simple angular dependence obtained for $\tau$ in Eq. (8) breaks down (see the green curve in Fig. 4). While the zero-torque points along the principal directions of magnetic anisotropy are maintained, the shape of the curve changes. Two different features emerge compared to the low field case: a smoother dependence of $\tau$ near the easy zero and a more abrupt change in $\tau$ near the hard zero. A detailed explanation of this behaviour is given in Appendix B, while here we must point out that this peculiarity is the key to disentangle individual contributions from noncollinear anisotropies in crystals. To highlight this effect, in Fig. 5 we plotted the magnetic torque (red lines) resulting from two orthogonal contributions to anisotropy (blue and green lines) in the low and high field limits. The sum of two orthogonal contributions is identically zero in the low field limit (when Eq. (8) holds) while it is not in the high field limit. The effects of noncollinearity, clearly visible in the shape of the red line of Fig. 5b, are a decrease in the signal and an increase in the number of zero-torque angles. The simple example reported in Fig. 5 can be easily extended to the case of more than two noncollinear contributions, evidencing the possibility to study complicated anisotropic architectures.

\subsection{Experimental set-up}

Several experimental set-ups are dedicated to measure magnetic torque. The detection of the torque moment can be for example optical [68-70], piezoelectric [71,72] or piezoresistive [73-75]. The combination of a cantilever with a magnetic tip allowed even the detection of a single spin [76]. In this review, we will instead focus on capacitive detection: a capacitive (electrical) detection of the deflection of the upper plate of a capacitor (the cantilever) requires an extremely simple and relatively inexpensive experimental set-up, but at the same time assures high sensitivity and fast data acquiring time $[77,78]$.

The capacitance $(C)$ of an ideal parallel plates capacitor is given by

$C=\frac{\varepsilon_{D} A}{d}$

\footnotetext{
4 This is a common scenario in the description of the magnetic anisotropy of $\mathrm{f}$ elements. High-order anisotropies are described in Section 5.2.
} 
where $\varepsilon_{D}$ is the permittivity of the dielectric that separates the two plates, $A$ is the area of the upper plate and $d$ is the separation between plates. The capacitance without any applied force is $C_{0}=\varepsilon_{D} A / d_{0}$. If we assume to have small deflections of the upper plate (linear regime), we can expand in series the capacitance, obtaining the difference $\Delta C$ as follows:

$\Delta C=C\left(d_{0}+\delta d\right)-C_{0}=-\frac{C_{0}}{d_{0}} \Delta d+\frac{C_{0}}{d_{0}^{2}} \Delta d^{2}-\frac{C_{0}}{d_{0}^{3}} \Delta d^{3}+\ldots$

Eq. (10) shows that the difference in capacitance is directly proportional to the deflection $(\Delta d)$ that is in turn proportional to the torque. The proportionality between these two quantities is clearly dependent on the design and material used to fabricate the cantilever and can be obtained after a proper calibration (see after).

The only required electronics are a capacitive bridge and a mechanical gear wheel to rotate along an axis perpendicular to the applied magnetic field. The core of the instrument is the capacitor sketched in Fig. 6, that has a fixed copper base plate (thickness $\approx 300 \mathrm{~nm}$ ) separated by $\approx 0.1 \mathrm{~mm}$ from an upper plate (the cantilever) that can be deflected elastically if a torque is acting on the sample that is fixed on top of it. The upper plate has a thickness of $\approx 25 \mu \mathrm{m}$ and it is usually made of copper-beryllium alloy ( $2 \%$ of Be). The choice of this particular mixture has several advantages with respect to other metallic alloys: it is diamagnetic, it has a linear expansion coefficient almost independent on temperature and it has high elastic and fatigue strengths [79]. The support and the spacer are made of Epoxy and have the function to establish a well-defined parallelism and distance $(\approx 100 \mu \mathrm{m})$ between the two plates. The gear wheel is connected to the core and allows a complete rotation around an axis ( $Y$ in Fig. $6 \mathrm{~b}$ ). The instrumental factor that relates the change in capacitance (distance) and the consequent torque can be simply obtained by applying a voltage to a loop formed by a gold wire fixed on the cantilever. In this way, a known magnetic moment is generated and the response of the instrument can be properly calibrated. Alternatively, an indicative scaling factor can be obtained measuring a crystal with wellknown weight and composition.

The sample can be either a single crystal or a thin film deposited on a support of any inert and diamagnetic material. In the case of single crystals, the sample is fixed on the cantilever with glue or grease. Of course, the orientation of the crystal should be known with great accuracy to define the mutual orientation of the laboratory $(X Y Z)$ and crystallographic $\left(a b^{\prime} c^{*}\right)$ reference frames (see Appendix C). Since the crystal is often visually aligned under a microscope, this is the biggest source of error in the measurement (usually between $1^{\circ}$ and $5^{\circ}$ ). In the following sections, we describe the principal information that we can extract using this technique.

\section{Orientation of magnetic anisotropy}

In this Section, we point out that the main utility of CTM is to detect the orientation of the magnetic anisotropy. The detection
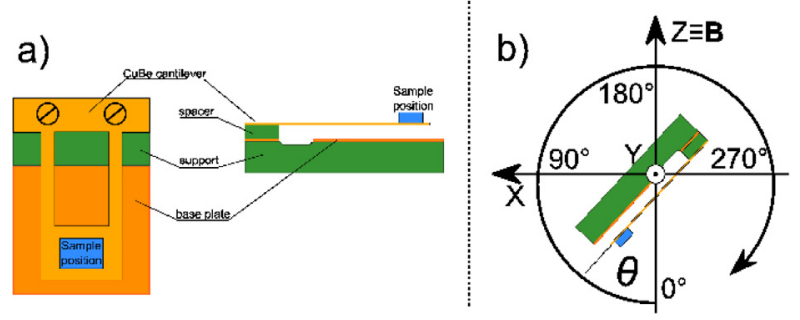

Fig. 6. (a) Top (left) and side (right) view of the main part of a capacitive torque meter. (b) Basic operation mode of the instrument, with rotation angle $(\theta)$ and laboratory $X Y Z$ reference frame on it. is quite easy if all the anisotropies are collinear, while it becomes tricky for complicated noncollinear architectures. To facilitate the reader, we divided this section in three parts: collinearity, intermolecular noncollinearity and intramolecular noncollinearity.

\subsection{Collinearity}

If all the molecular anisotropies inside a crystal are collinear, CTM provides a unique solution for the orientation of the magnetic anisotropy. The structures that exhibit true collinearity are the easiest to study, but are also quite rare and often belonging to the triclinic crystal system. To circumvent this problem, several papers that exploit CTM to find or confirm the orientation of magnetic anisotropy, are based on giant spins originated in polymetallic clusters [80,81]. Examples of strictly mononuclear [82] or almost dimeric [83] complexes are rarer due to the widespread use of SCM to characterize collinear systems [22].

A textbook example is LnTRENSAL [84,85], whose structure was already analysed in Section 2.1 and reported in Fig. 1. The isostructural lanthanide derivatives of this molecule were studied both from a magnetic [86] and a spectroscopic [86-88] point of view, to obtain the Crystal Field (CF) parameters and to investigate the strong interaction between this molecule and a graphene or a $\mathrm{Ru}$ (0001) surface $[89,90]$. Before discussing the results obtained on three derivatives of this family that were studied using CTM ( $\mathrm{Ln}=\mathrm{Tb}, \mathrm{Dy}$ and $\mathrm{Er}$ ), we must remark that the assumption of collinearity is justified by the negligible anisotropy in the $a b$ crystallographic plane (see ESI of Ref. [82]). Thus, the most relevant plane to be scanned in these systems contains the $c$ axis (the $C_{3}$ symmetry axis of the trigonal space group). In Fig. 7, we reported the experimental results obtained using a field of $12 \mathrm{~T}$ at $T=5 \mathrm{~K}$ for the three studied derivatives.

For these systems, the sign of torque unequivocally determines the nature of magnetic anisotropy. The upper panel of Fig. 7 describes the relation between sign of torque and magnetic

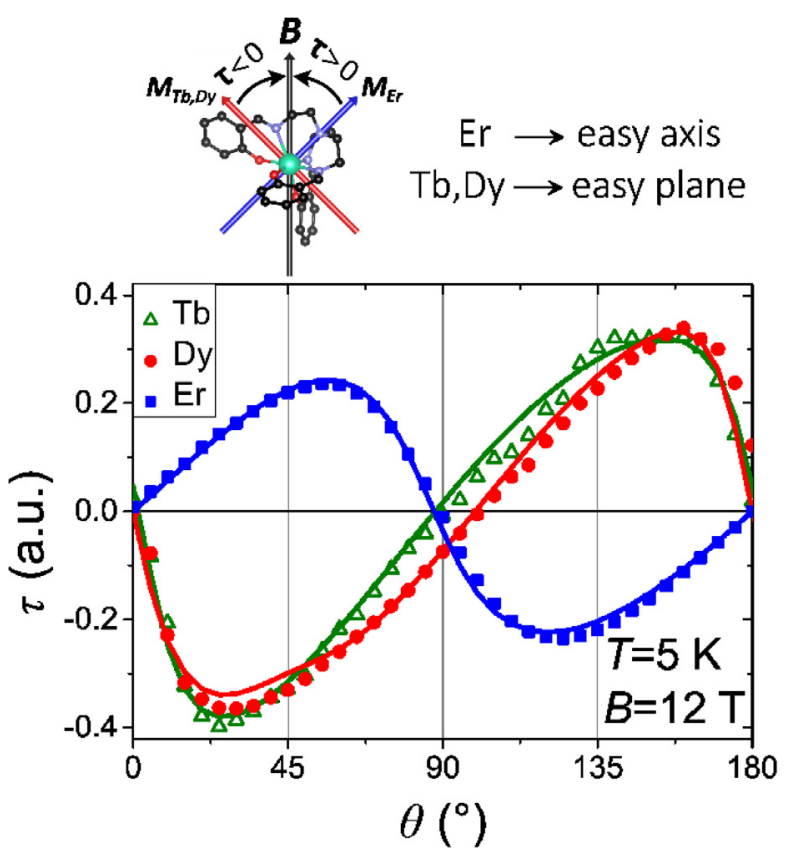

Fig. 7. Torque signals for TbTRENSAL (empty green triangles), DyTRENSAL (full red circles) and ErTRENSAL (full blue squares) recorded at $T=5 \mathrm{~K}$ and $B=12 \mathrm{~T}$. The solid lines represent the best fit (see text). At $\theta=0^{\circ}$ the field was aligned parallel to $c$. The upper panel shows the relation between sign of torque and magnetic anisotropy at $\theta=45^{\circ}$. Following the convention adopted by the author, a positive torque points towards the reader. 
anisotropy at an arbitrary chosen angle $\left(\theta=45^{\circ}\right)$. At this angle, a positive torque implies that the $c$ crystallographic axis is the easy magnetization axis while a negative torque suggests the opposite (we remark here that these are the only two possibilities compatible with the ligand geometry). It is then clear that ErTRENSAL has an easy axis anisotropy while DyTRENSAL and TbTRENSAL possess an easy plane anisotropy. An equivalent way to define the sign of magnetic anisotropy in these complexes is to look at the slope of the curves. Around $\theta=0^{\circ}(\mathbf{B} / / c)$ TbTRENSAL and DyTRENSAL exhibit a rapid angular variation of $\tau$ (hard zero) while for ErTRENSAL the slope is less pronounced (easy zero). Opposite considerations can be done around $\theta=90^{\circ}$. It is also interesting to notice that the torque curve of DyTRENSAL reported in Fig. 7 does not exhibit the $90^{\circ}$ periodicity extensively discussed in Section 3.1. This effect, only visible at low temperature and high magnetic field (see ESI of Ref. [82]) can be described using high-order CF operators (see Section 5.2). These results, in agreement with previous findings [86], confirm that CTM is extremely sensitive to orientation and nature of magnetic anisotropy of molecular species.

\subsection{Intermolecular noncollinearity}

The literature reports on intermolecular collinearity solved using CTM are limited [91-93] due to the crescent complexity of the systems. An easily understandable proof of concept on how to disentangle noncollinear contributions arising from different molecules in the unit cell was reported few years ago [91]. The studied system was an $\operatorname{Er}(\mathrm{III})$ mononuclear organometallic complex called $\mathrm{Cp}^{*}$ ErCOT $\left(\mathrm{Cp}^{*}=\right.$ pentamethylcyclopentadiene anion and COT $=$ cyclooctatetraene dianion) $[94,95]$. The molecule crystallizes in the orthorhombic Pnma space group, where only two noncollinear families of molecules are present, due to the presence of an inversion centre in the middle of the cell and a mirror plane inside the molecule. As reported in Fig. 8, the noncollinear molecules inside the cell are almost orthogonal to each other. Although this represents a complicated scenario concerning the study of noncollinearity, this system was previously studied using out-ofequilibrium SCM measurements, exploiting the hysteretic behaviour below $5 \mathrm{~K}$ [96]. This study was based on a combination of experimental results and $a b$ initio calculations. The main conclusion was that the anisotropy was axial, with the $z$ axis almost perpendicular to the plane of the organic rings.

The simulation reported in Fig. 5b suggested that the magnetic torque produced by two orthogonal molecules is not zero. The shape and angular dependence of the torque curves is instead related to the magnetic structure of the crystal. The experimental magnetic torque obtained rotating the crystal along the $b$ axis reported Fig. 9 is the experimental proof. All the curves recorded at different applied magnetic fields collapse to zero at $\theta=42^{\circ}$ and $\theta=42+90=132^{\circ}$, corresponding to B along $a$ and along $c$, respectively. ${ }^{5}$ Although no additional features are present in the curve registered at $B=1 \mathrm{~T}$, when the field is raised the curves become more structured and the torque vanishes at other two angles $(\theta=98$ $109^{\circ}$ and $\theta=152-163^{\circ}$, dashed lines in Fig. 9). Noticeably, these two additional zero-torque angles are dependent on the magnetic field, and their position is strongly connected to the shape of the magnetic anisotropy.

After the fitting of the experimental curves (in Ref. [91] two different types of fit were performed), the orientation of the magnetic anisotropy was obtained. It is important to remark that the sign of the anisotropy was determined without ambiguity. Although the relative position of the anisotropies of the two molecules in the

\footnotetext{
${ }^{5}$ In the orthorhombic crystal system, the main crystallographic axes must be also the main magnetic axes of the crystal susceptibility tensor, so when the field is parallel to $a, b$ or $c$ the torque must vanish.
}

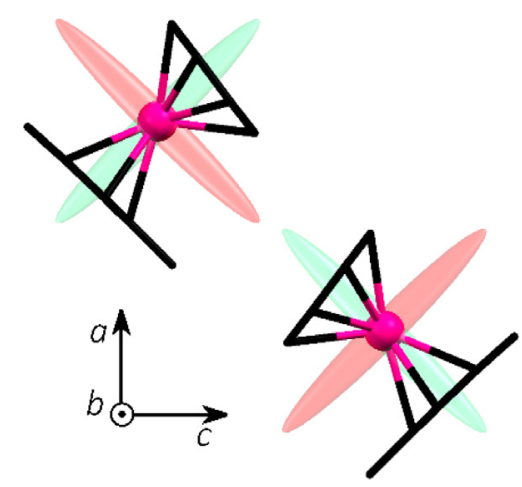

Fig. 8. Magnetically inequivalent molecules in the Cp*ErCOT unit cell. Red and green ellipsoids represent the two solutions obtained via CTM (green is the correct one). The long axis of the ellipsoids is the $z$ (easy) axis and the $x y$ plane was magnified for clarity. Colour code: Er-pink, C-black (H omitted).

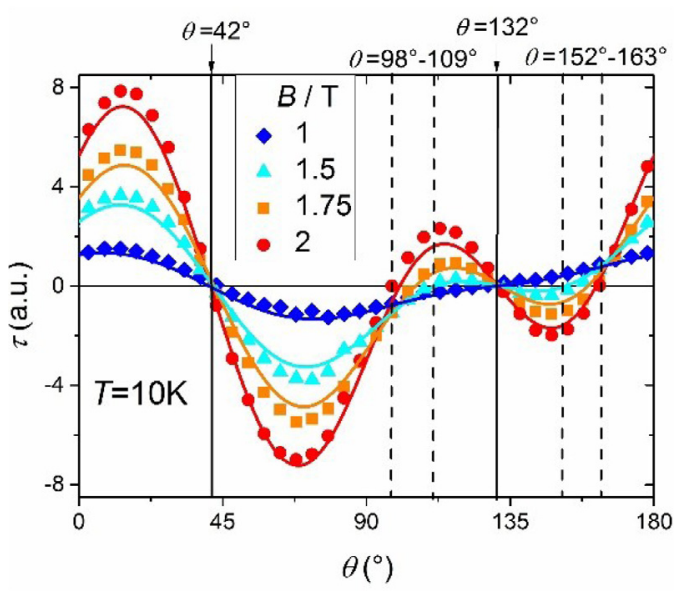

Fig. 9. Angular dependence of the magnetic torque of a single crystal of $\mathrm{Cp}^{*} \mathrm{E}$ rCOT measured at $T=10 \mathrm{~K}$ and various applied fields. Solid lines correspond to the simulation using an effective $S=1 / 2$ (see Ref. [91]).

unit cell is fixed and known, it is in principle possible to assign the obtained solution to both molecules (red and green ellipsoids in Fig. 8). Alternatively, we can consider only one molecule, with two possible solutions provided by the fit (leading to the same conclusion). In this case both the symmetry of the system (pseudo $C_{\infty}$ axes perpendicular to the rings) and the previously reported results [96], clearly pointed out that the correct solution was the green one in Fig. 8.

It is important to remark that it was possible to find the correct solution for $\mathrm{Cp}^{*} \mathrm{ErCOT}$ only due to the previously reported theoretical calculations. For noncollinear systems torque must be used in combination with theoretical calculations, as in Refs. [92,93]. On the other hand, we must stress that CTM does not require the presence of hysteresis to detect a signal coming from noncollinear anisotropies, thus in this respect it can be considered much more generally applicable than SCM.

\subsection{Intramolecular noncollinearity}

The determination of intramolecular noncollinearity is clearly one of the most interesting uses of CTM because it allows to isolate the anisotropy of the single building blocks used to create a given chemical structure. However, the presence of exchange interaction can complicate the treatment of data [45,97]. To overcome this problem, a giant spin approximation $[98,99]$ or molecules with poorly coupled centres [100] can be useful. Despite the great 
variety of systems studied using CTM, in this review we limit the discussion to two textbook examples.

A clarifying example is the CTM investigation of a trigonal crystal (space group $R-3 c$ ) of $\mathrm{Fe}_{3} \mathrm{La}$ [45]. The diamagnetic $\mathrm{La}$ (III) ion at the centre of the molecule, shown in Fig. 2, hampers exchange interactions between the central metal and the peripheral Fe(III) ions, typical of the $\mathrm{Fe}_{4}$ family of SMM. For this reason, the giant spin approximation is not valid, providing access to the single ion anisotropy.

Fig. 10 shows the angular dependence of the torque recorded at $T=2.3 \mathrm{~K}$ and $B=3 \mathrm{~T}$ for two different rotations (Rot1 and Rot2). The inset of Fig. 10 shows the relation between the crystallographic reference frame and the rotations performed during the experiment. Except the trivial $180^{\circ}$ periodicity typical of torque of paramagnetic anisotropic molecules, Rot 1 does not display any additional symmetry property. This is expected when the free energy is scanned in a plane perpendicular to the $b$ crystallographic axis, as reported in Fig. 10, top. Moreover, the zeroes of the rotation are clearly not evenly spaced (meaning that both Eq. (8) and the Giant Spin approximation are not valid and the resultant torque can indeed provide information on the single atom anisotropy). On the other side, Rot2 is antisymmetric with respect to $\theta=0^{\circ}$ because the scanned plane contains the $b$ axis (a $C_{2}$ molecular axis). The strategy that the authors propose for the fit of data is based on iterative fits including more parameters at each step. Neglecting scaling factors and offsets, the final fit included four relevant parameters, namely the axial $\left(D=0.989(9) \mathrm{cm}^{-1}\right)$ and the rhombic $\left(E=0.0517 \mathrm{~cm}^{-1}\right.$ ) Zero Field Splitting (ZFS) parameters, the angle between the crystallographic $c$ axis and the local $z$ axes $\left(\xi=68.89(8)^{\circ}\right)$ and the coupling constant $\left(J=0.0783(19) \mathrm{cm}^{-1}\right)$. An $E / D$ ratio as small as 0.05 testify an almost axial easy plane anisotropy for each metal site (see also Eq. (11)). The angle $\xi$ (the only relevant Euler angle since the intermediate axis of the first tensor was fixed by the authors along the $b$ crystallographic axis, in agreement with previous works [26,101-103]) arranges the anisotropy tensors in such a way that the $z$ axis of the first tensor is almost directed along a $\mathrm{O}-\mathrm{Fe}-\mathrm{O}$ bond (see Fig. 2). Finally, the low value of the coupling constant confirms a posteriori the effectiveness of the central $\mathrm{La}$ (III) ion in hampering the interactions between $\mathrm{Fe}$ (III) ions. Interestingly, this work unravelled that the easy axis
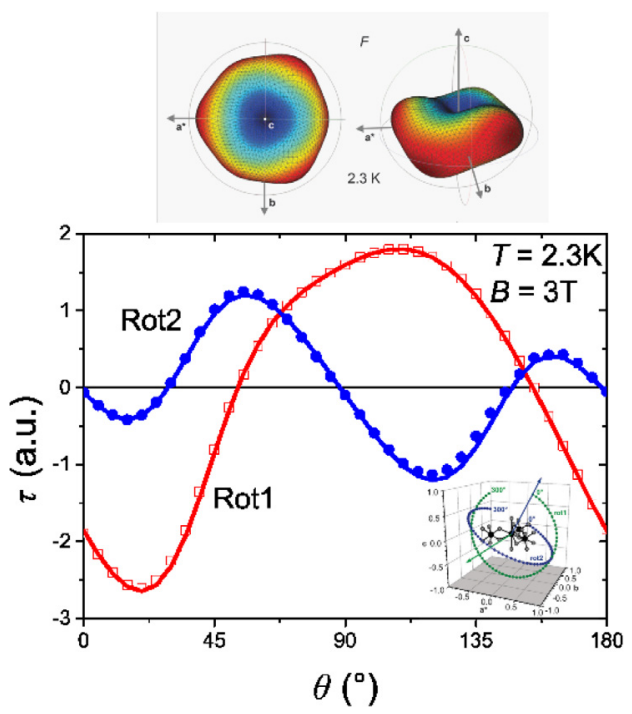

Fig. 10. Top: Free Energy surface of $\mathrm{Fe}_{3} \mathrm{La}$ calculated at $T=2.3 \mathrm{~K}$. Bottom: Angular dependence of magnetic torque for $\mathrm{Fe}_{3} \mathrm{La}$ recorded at $T=2.3 \mathrm{~K}$ and $B=3 \mathrm{~T}$ (full dots and empty squares). Solid lines represent the best fit. The inset shows the planes scanned during the two rotations.
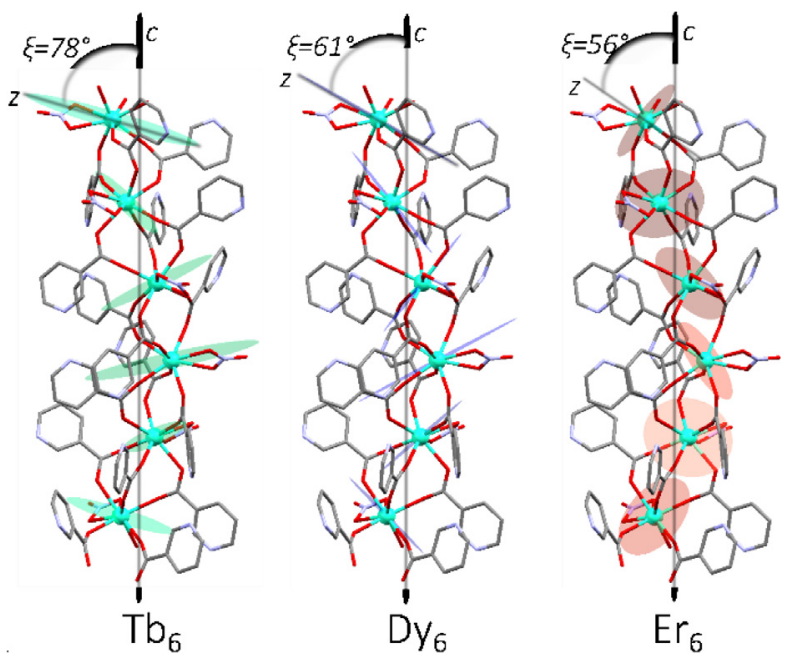

Fig. 11. Susceptibility tensors calculated at $T=5 \mathrm{~K}$ and $B=0.1 \mathrm{mT}$, plotted on the molecular structure. The $x y$ plane for $\mathrm{Dy}_{6}$ was magnified by a factor 10 to give a more solid aspect to the ellipsoids.

magnetic anisotropy that characterizes the $\mathrm{Fe}_{4}$ derivatives is not originated by a sum of easy axis local tensors. Instead, the only the central Fe(III) ion has a negative ZFS [104], while the peripheral ions have an easy plane anisotropy with the hard axis tilted by almost $70^{\circ}$ from the molecular easy axis.

A second example of intramolecular noncollinearity studied via CTM is the spin helicity in chiral chains [100]. The structure of the studied complex, chemically described in Refs. [105,106], is reported in Fig. 11. The generic formula $\left[\mathrm{Ln}(\mathrm{Hnic})(\mathrm{nic})_{2}\left(\mathrm{NO}_{3}\right)\right]_{\mathrm{n}}$ (where Hnic is the nicotinic acid) was obtained for $\operatorname{Ln}=\mathrm{Tb}$, Dy and Er. Since the helical structure crystallizes in the hexagonal enantiomeric $P 6_{1} / P 6_{5}$ space groups, the repeating unit is formed by 6 metal ions with identical coordination environment ( 8 oxygen atoms). To facilitate the reader, the $\mathrm{Dy}, \mathrm{Tb}$ and $\mathrm{Er}$ derivatives will be hereafter called $\mathrm{Dy}_{6}, \mathrm{~Tb}_{6}$ and $\mathrm{Er}_{6}$, respectively. To map the single ion anisotropies of this structure the authors performed two rotations: Rot1 (along the chain axis that is the $c$ crystallographic axis) and Rot2 (from the $c$ axis to the $a b$ plane). As a representative example, in Fig. 12 we reported Rot1 (a) and Rot2 (b) for Dy ${ }_{6}$ taken at $T=2 \mathrm{~K}$ and $B=7 \mathrm{~T}$ plotted using polar coordinates $(\theta=$ rotation angle, $r=|\tau|$ ). The experimental data were fitted using a model that considered six uncoupled metal centres related by a $\mathrm{C}_{6}$ axis (black solid line).

As expected, the two rotations have a completely different shape. In Rot 1 , the torque vanishes every $30^{\circ}$ but the overall symmetry of the polar curve is not $\mathrm{C}_{12}$ but $\mathrm{C}_{6}$ : this is the distinctive feature that the measurements were taken in the regime in which $\mathrm{Eq}$. (8) is not valid (see Appendix B for further details). We can also distinguish the two different zero points, already defined in Section 3.1. At $\theta=30+60^{*} k^{\circ}(k=0 \ldots 6$, integer $)$ torque goes to zero because $B$ is parallel to the projection of the easy axis in the plane of rotation for two ions and the other four are cancelling out in couples. Conversely, at $\theta=0+60 * k$ the zeroes are originated by the fact that $B$ lies in the $x y$ plane of two ions and the other four are again eliding by symmetry. It is important to notice that since the signal during Rot 1 is relatively strong (comparable to Rot2), we can already exclude the possibility that the chain is formed by collinear anisotropies with the principal axis along the chain axis. The angular dependence of Rot2 is considerably more complex, with a $C_{2}$ symmetry. Despite the trivial $0+90^{*} k^{\circ}(k=1$ $\ldots 4$, integer) zeroes ( $B$ parallel or perpendicular to the chain axis), Rot2 exhibits other zeroes at ca. $30^{\circ}, 150^{\circ}, 210^{\circ}$ and $330^{\circ}$. The presence of these zeroes is the proof that the anisotropy has a signifi- 

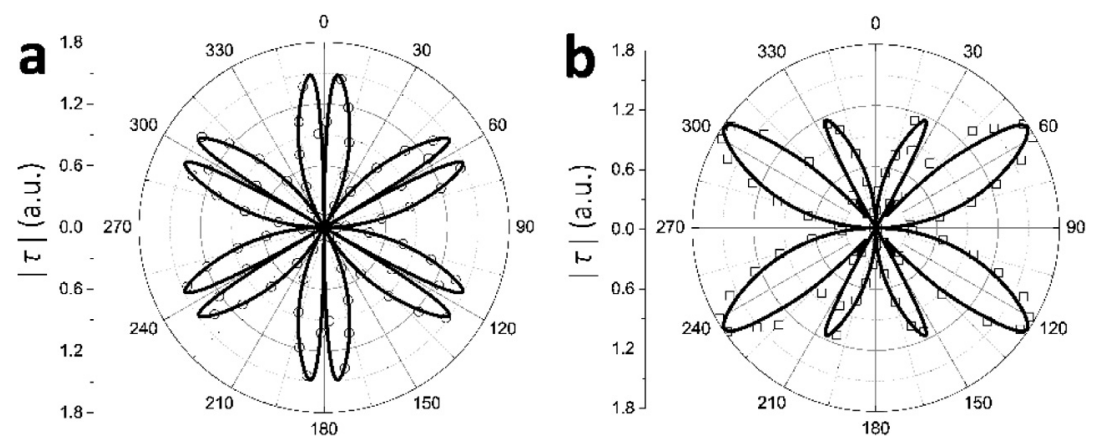

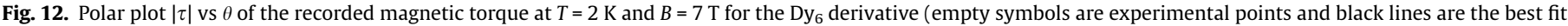
curves). (a) Rot1 (in plane), (b) Rot2 (from axis to plane).

cant component in the $a b$ plane, meaning that the chain exhibits spin helicity.

The fit of the experimental data was performed using five relevant parameters: three Euler angles $(\rho, \xi$ and $\psi)$ and two CF parameters to define axiality and rhombicity of the tensors $\left(b_{2}^{0}\right.$ and $b_{2}^{2}$, respectively). For more information about this notation see also Section 5.2. The use of just two CF parameters to describe these complex systems must be considered purely phenomenological, being only able to provide the sign of anisotropy (easy axis or easy plane) and the presence or absence of rhombicity. Moreover, we stress again that CTM provides six possible solutions for the orientation of the magnetic anisotropy of this system. However, the most relevant information is provided by the $\xi$ Euler angle, that is defined as the tilting angle between the chain axis and the $z$ molecular axis of the susceptibility tensors. ${ }^{6}$ In Fig. 11 the susceptibility tensors obtained as one possible solution were superimposed to the chemical structure. Both $\mathrm{Dy}_{6}$ and $\mathrm{Er}_{6}$ are extremely axial (easy axis and easy plane, respectively), while $\mathrm{Tb}_{6}$ is strongly rhombic. All the three values of $\zeta$ are higher than the magic angle ${ }^{7}$; this implies that the chain axis is the molecular hard axis for $\mathrm{Tb}_{6}$ and $\mathrm{Dy}_{6}$ but is instead an easy molecular axis for $\mathrm{Er}_{6}$. These conclusions were supported by standard magnetization measurements performed on oriented single crystals. Moreover, only $\mathrm{Dy}_{6}$ derivative shows slow relaxation of the magnetization, as expected for a strongly axial compound [100]. As a final remark, we notice that the anisotropy of these derivatives is opposite compared to the LnTRENSAL family, remarking the easy tunability of magnetic anisotropy by chemical design.

\section{Determination of the energy levels splitting}

The determination of the CF splitting is one of the most relevant topics in magnetochemistry. The high tunability of the number and nature of the ligand's atoms, as well as the internuclear distances allowed the synthesis of a huge number of compounds with relevant magnetic properties. Hereafter, we will describe how CTM can give access to the strength of the energy levels splitting originated by the presence of the ligands. The Section is divided in two subsections due to the different approach used to determine this splitting.

\subsection{Breaking field method}

The determination of the ZFS of Transition Metal (TM) ions is commonly performed using different techniques ranging from EPR to DC magnetometry. The two main parameters that are

\footnotetext{
${ }^{6}$ The angle $\zeta$ is unique for all the tensors.

7 The angle at which the dipolar interactions are zero, 54.74。
}

connected to ZFS are $D$ (axial parameter) and $E$ (rhombic parameter). The ZFS Hamiltonian can be written as follows:

$\mathcal{H}_{\text {ZFS }}=D\left(\widehat{\mathbf{S}}_{z}-\frac{1}{3} \widehat{\mathbf{S}}^{2}\right)+E\left(\widehat{\mathbf{S}}_{x}^{2}-\widehat{\mathbf{S}}_{y}^{2}\right)$

Following the formalism of Eq. (11), if $E=0$ the system is perfectly uniaxial with easy axis $(D<0)$ or easy plane $(D>0)$ anisotropy. The value of $E$ is instead connected to a difference in the hard or easy plane, thus the ratio $E / D$ quantifies the degree of rhombicity of the system. In rare cases, also higher order parameters are included in the description [10]. If the investigated system can be satisfactorily described with these parameters, the most widespread approach to quantify them is to perform Breaking Field measurements.

Since the magnetic torque of an isotropic paramagnetic is identically zero, one can think that for extremely high fields and low temperatures the magnetic torque goes to zero due to the saturation of the magnetization. In Fig. 13 we simulated the $\tau$ vs $B$ curves of a mole of $\mathrm{Mn}_{12}$ complex [63-66]. For high fields, all the curves tend to a finite value. It was already shown [107], that for an Ising system it reaches the limiting value of

$\lim _{B \rightarrow \infty} \tau_{y}=-2 D S\left(S-\frac{1}{2}\right) \sin \theta \cos \theta$

where $S$ is the total spin of the atom and $D$ is the ZFS parameter. For angles close to the hard zero (red curve in Fig. 13), the intensity of torque versus field reaches a maximum. The field that corresponds to this point is called "breaking field": the minimum field able to overcome the anisotropy of the molecule, forcing $\mathbf{M}$ to tilt towards an unfavourable direction. Clearly, the position of the maximum is very sensitive to the magnitude of the ZFS parameter and on the position of the molecular reference frame with respect to the magnetic field. For this last reason, these measurements require to optimize with high precision the position of the sample, normally recording $\tau$ vs $B$ curves at two angles close and symmetric to $90^{\circ}$ (e.g. $91^{\circ}$ and $89^{\circ}$ ) and subtracting them [99].

An excellent example of how to apply this approach is reported in Ref. [99], for the $\mathrm{Mn}_{12}$ cluster [108]. Assuming a giant spin approximation, the AFM coupling between the four Mn(IV) and the eight $\mathrm{Mn}(\mathrm{III})$ ions in the cluster produces a spin $S=10$ ground state. The fourth order axis of the tetragonal crystal group I-4 passing through the centre of the molecule assures collinearity, so the crystal was positioned with the easy axis (along the $c$ crystallographic axis) almost perpendicular to the applied field. The red curve in Fig. 14, that well reproduces both the shape and the saturation value, was simulated using an axial $g$ tensor $\left(g_{\text {par }}=1.93\right.$, $\left.g_{\text {perp }}=1.96\right)$ and two axial ZFS parameters $\left(D=-0.468 \mathrm{~cm}^{-1}\right.$ and $\left.b_{4}^{0}=-2.4 \cdot 10^{-5}\right)$. To get insight into the sensitivity of CTM, in Fig. 14 we simulated the same curve neglecting the $b_{4}^{0}$ (green curve) and using an isotropic $g=2$ (blue curve). It appears clear 


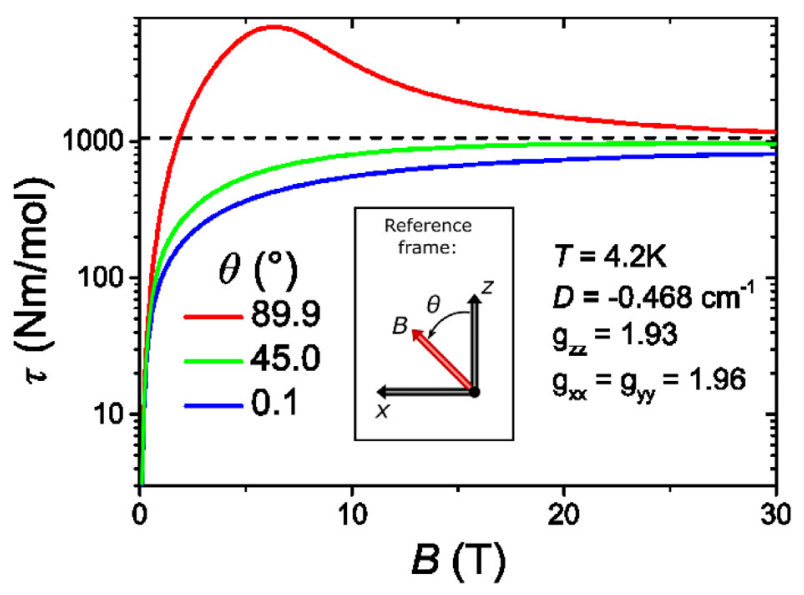

Fig. 13. Simulated field-dependence of the magnetic torque for a mole of a $\mathrm{Mn}_{12}$ complex with $D=-0.468 \mathrm{~cm}^{-1}$ at three different angles between the magnetic field and the easy axis. All the curves were divided by a factor $\sin \theta \cos \theta$ to emphasize that they tend to the same saturation value ( $1064 \mathrm{Nm} / \mathrm{mol}$, dotted line).

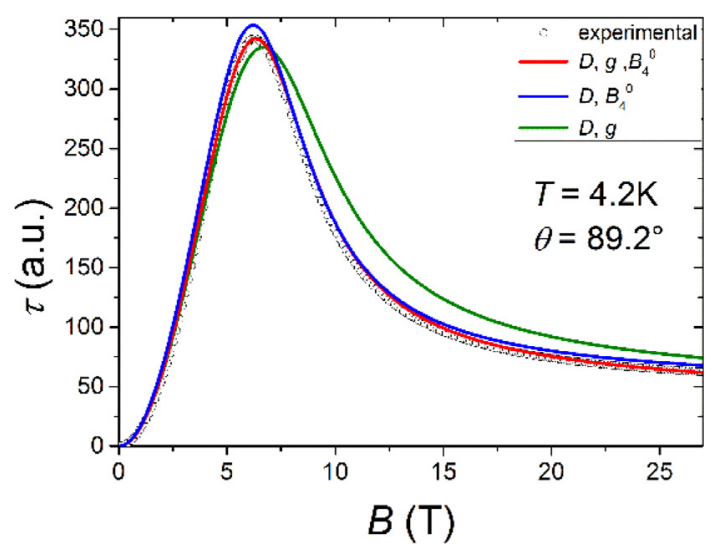

Fig. 14. Field dependence of the torque signal of a crystal of $\mathrm{Mn}_{12}$ at $T=4.2 \mathrm{~K}$ and $\theta=89.2^{\circ}$ (empty circles, data from Ref. [99]). Solid lines are simulations, multiplied by a scaling factor, obtained using different sets of parameters (see text).

that the fourth order parameter has a strong influence on both the shape of the curve and on the position of the maximum, conversely CTM is less sensitive to small changes in the $g$ tensor. This poor sensitivity to $g$ was also recently found in the cationic pentacoordinate $\mathrm{M}\left[\mathrm{Me}_{4}(\text { cyclam }) \mathrm{N}_{3}\right]^{+}\left(\mathrm{M}=\mathrm{Co}\right.$ or $\mathrm{Ni} ; \mathrm{Me}_{4}($ cyclam $)=$ tetramethylcyclam; $\mathrm{N}_{3}=$ azido)complexes [93].

Another intriguing effect related to this kind of measurements was observed on a Mn(II)-[3 $\times 3$ ] grid by Waldmann et al. [58]. They measured a change in sign of the magnetic torque as a function of the intensity of the magnetic field. This effect was attributed to a level crossing between levels with opposite sign of the ZFS parameters. In other words, they observed a change in anisotropy from easy axis to easy plane. Indeed, we have already pointed out in Section 4.1 that the sign of torque in collinear systems is directly related to the anisotropy shape. More importantly, this result demonstrates that torque can be used as a quasi-spectroscopical method, providing access to information, such as levels crossings, that are typically seen only with spectroscopic techniques. As a final remark on this paragraph, we recall that the breaking field approach is suitable to investigate slightly anisotropic transition metal complexes at low $T$ while for highly anisotropic complexes an approach based on the thermal population of the energy levels is required.

\subsection{Maxwell Boltzmann statistics}

A more general approach to extract the energy levels splitting and wavefunctions composition is to perform temperature dependent measurements. This is particularly relevant for Ln complexes because the inner nature of the partially filled $4 \mathrm{f}$-orbitals of Ln that prevents the quenching of the orbital angular momentum ( $L$ ), combined with the high coordination numbers (usually 8 or 9) requires a CF description that goes beyond the spin-only approximation [109]. Indeed, the most common way to describe the potential around the $\mathrm{Ln}$ is to use $\mathrm{CF}$ parameters with order up to 6 . Considering only the ground $J$ multiplet, ${ }^{8}$ the CF Hamiltonian can be conveniently described using the Stevens' extended operators as follows:

$\mathcal{H}_{C F}=\sum_{k=2,4,6} \omega(k) \sum_{q=-k}^{k} b_{k}^{q} \widehat{\mathbf{0}}_{k}^{q}$

where $\omega(k)$ is a parameter (different for each $f^{n}$ configuration and $k$ value) which accounts for the proportionality between the spherical harmonics of order $k$ and the corresponding operator equivalent for that configuration. It is common to rename $\omega(k)$ in three different ways as a function of $k$ as follows: $\omega(2)=\alpha, \omega(4)=\beta$ and $\omega(6)=\gamma$. The number and nature of the $\widehat{\boldsymbol{O}}_{k}^{q}$ operators is strictly dependent of the single site geometry and can be as high as 27 to describe a nonsymmetric system [109]. When one or more symmetry elements are present, some $\mathrm{CF}$ parameters vanish, thus reducing the number of independent parameters in the calculation or fit.

Usually, the energy levels splitting of the ground multiplet of Ln is of the order of several hundreds of $\mathrm{cm}^{-1}$ [12], thus an approach based on the determination of the high field limit of torque at low temperature is clearly unreliable because the high single-ion anisotropy shifts the Breaking Field value at very high fields (not achievable with common magnetometers). Moreover, we can also argue that the fit of a measurement performed at low $T$ is clearly not suitable to determine the wavefunction composition, due to the lack of population imposed by the Maxwell Boltzmann statistics.

The first seminal report on the possibility to determine the CF parameters of Ln using torque magnetometry was written by Kuz'min [110]. In this paper, the author proposed a choice for the optimum field and temperature range to reach the best sensitivity to certain CF parameters based on the value of the Langevin ratio ${ }^{9}$ [111]. As an example, for a $\mathrm{Tb}^{3+}$ ion at an optimum field of $B=2 \mathrm{~T}$, the best sensitivity to $b_{2}^{0}$ is reached in the interval $77-300 \mathrm{~K}$, for $b_{4}^{0}$ at $10-20 \mathrm{~K}$ and for $b_{6}^{0}$ at $4.2-10 \mathrm{~K}$.

As an example, we can quote again the paper on LnTRENSAL system, already discussed in Section 4.1. We remind here that ErTRENSAL possesses an easy axis anisotropy, while TbTRENSAL and DyTRENSAL have an easy plane anisotropy. The measurements on these crystals were taken in a range of temperature from $2 \mathrm{~K}$ to ca. $150 \mathrm{~K}$ (the upper limit was simply determined by the experimentally detectable signal). At each temperature, at least two rotations acquired at different $B$ were measured to increase the sensitivity of the technique to the composition of each state. The fit proposed by the authors was based on the eight relevant $\mathrm{CF}$ parameters that must be used to describe a $C_{3}$ symmetry. One has to keep in mind that the uncertainty on some parameters can be huge due to correlation between parameters, also observed for other techniques [87,89]. The energy levels splitting of the

\footnotetext{
$8 \mathrm{~J}$ is the total angular momentum of the ground state, obtained following a Hund's rule. If the outermost subshell is less than half-filled $J=|L-S|$, while for more than half-filled outermost subshells $J=L+S$.

${ }^{9}$ The Langevin ratio is defined as $g_{\mathrm{J}} \mu_{\mathrm{B}} J B / k_{\mathrm{B}} T$, thus it compares the strength of the Zeeman energy with the thermal energy.
} 


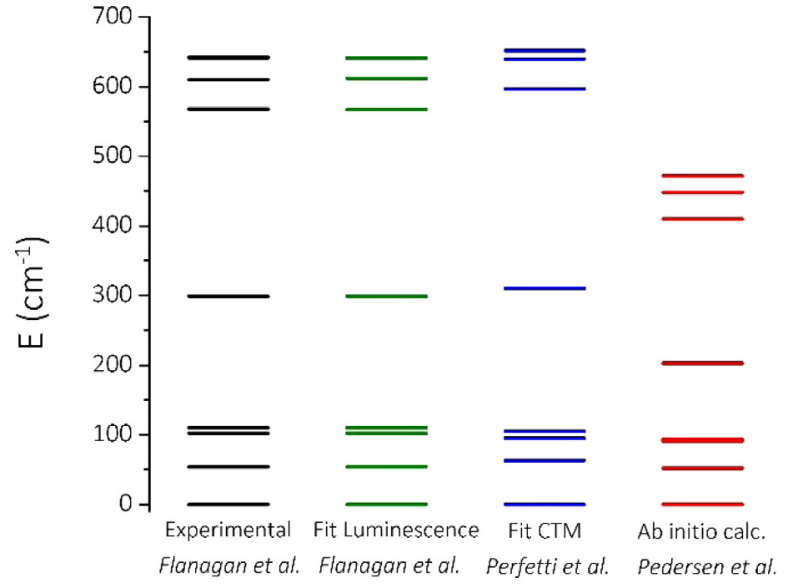

Fig. 15. Energy levels pattern of the ground ${ }^{4} \mathrm{I}_{15 / 2}$ multiplet of ErTRENSAL. BlackExperimental (Luminescence [88]), Green-Fit of Luminescence data [88], Blue-Fit of CTM data [82], Red- $A b$ initio calculation on a molecule embedded in 5 layers of point charges [89].

ground multiplet of ErTRENSAL is reported in Fig. 15. For this derivative spectroscopic data of the energies of the ground state were measured (black lines) and fitted by Flanagan et al. (green lines) [88]. As expected, the agreement is excellent. The set of parameters provided by CTM (blue lines) is also in good agreement with the spectroscopic study (the best agreement is found for the lowest four states that are the ones significantly populated in the investigated temperatures). Moreover, the extracted set of $\mathrm{CF}$ parameters was also used to correctly reproduce the temperature dependence of the magnetic susceptibility [82]. However, it is important to stress here that the initial guess of parameters is crucial, especially when the fitted CF parameters are $>2$. Conversely, the agreement between experimental and $a b$ initio calculations (red lines) is considerably good for the low-lying states but is rather poor for $E>100 \mathrm{~cm}^{-1}$. This picture enlightens that CTM can be a powerful experimental technique to obtain the CF splitting of highly anisotropic complexes, especially when flanked by spectroscopic techniques.

\section{Magnetization steps and hysteresis curves}

Magnetization curves characterized by sharp steps are among the most intuitive and fascinating experimental proofs that the energy of a molecule is quantized [10]. Due to the aforementioned relation between torque and magnetization, the appearance of steps in the $\tau$ vs $B$ graph must be expected. The first experimental evidence was provided in 1998 by Perenboom et al. using single crystal of $\mathrm{Mn}_{12}$ [112,113]. After this, several papers were published on the observation of similar steps in molecular rings [52,80,114,115] and on magnetic dimers [83]. In Fig. 16 we reported a comparison between a magnetization curve recorded on a $\mathrm{Fe}_{10}$ molecular ring at $T=0.6 \mathrm{~K}$ [57] and the capacitance variation of a cantilever torque meter at $T=0.45 \mathrm{~K}[80]$. Although the small temperature difference precludes a straightforward comparison, the agreement between the position of the step is remarkably good. Interestingly, the capacity (or equivalently the torque) signal between two steps is not flat. It was demonstrated that this feature is characteristic of the mixing between states [52,116].

Another interesting case exhibiting step-like torque curves is represented by a sodium cation trapped inside a six-membered ferric wheel ( $\mathrm{Na}_{\mathrm{Fe}} \mathrm{F}_{6}$ [52]. The axial symmetry of the complex is $\mathrm{S}_{6}$ and the ground state was initially expected to be a pure singlet due to the antiferromagnetic coupling between Fe ions. The mag-

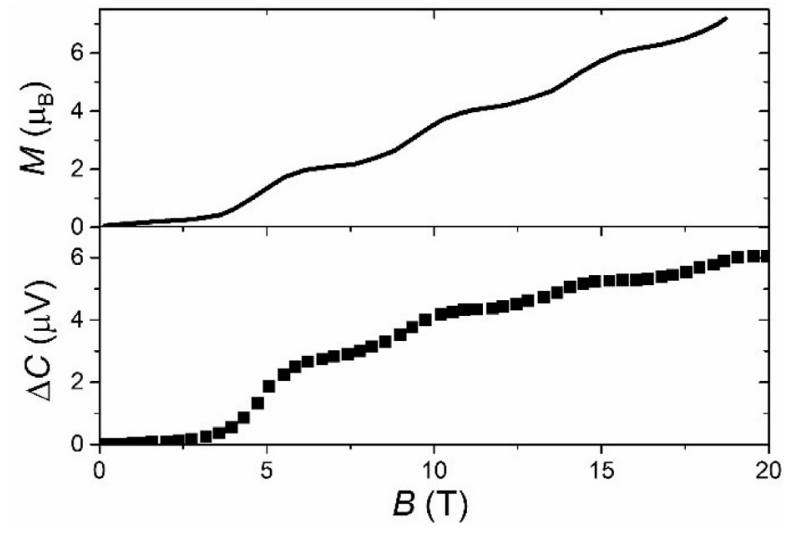

Fig. 16. Comparison between magnetization curve (solid line, $T=0.6 \mathrm{~K}$, Ref. [57]) and capacitance variation (black squares, $T=0.45 \mathrm{~K}$, Ref. [80]) recorded on a $\mathrm{Fe}_{10}$ molecular ring.

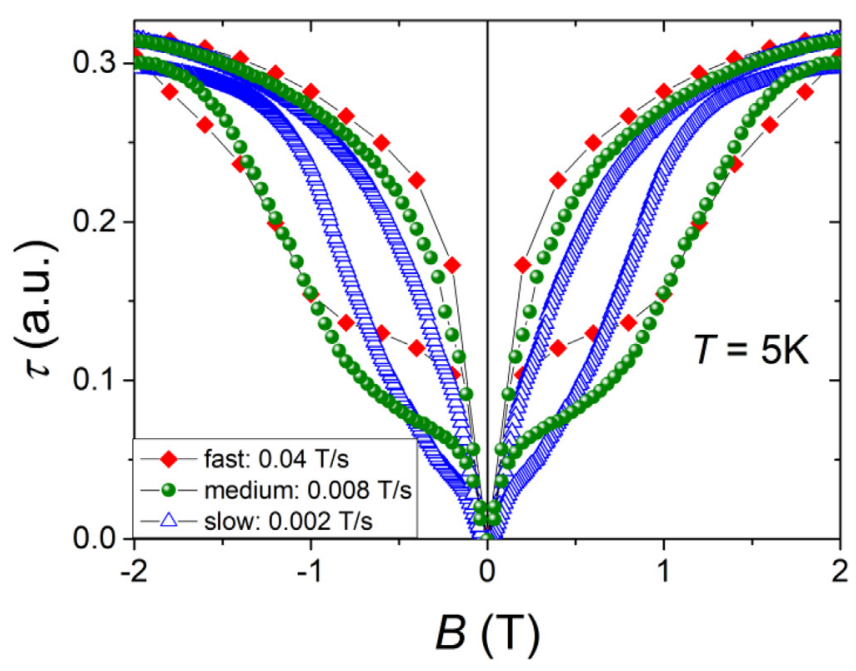

Fig. 17. Hysteresis curves measured on $\mathrm{Cp} * \mathrm{ErCOT}$ single crystal at different field sweep rates [91].

netic torque of this compound measured at $220 \mathrm{mK}$ exhibits a huge step when the applied field is increased from 16 to $17 \mathrm{~T}$. The positive slope of the torque signal before the step and the finite width of the step itself clearly excluded that the ground state of the wheel could be described as a pure singlet. The solution proposed by the authors in order to reproduce the experimental evidences, is to lower the symmetry of the complex to $C_{3}$ and describe the Hamiltonian of this system as composed by a Zeeman term plus an interaction term analogous to the one reported in Eq. (1), but expanded for all the pairs in the wheel. The key point of this model is that the introduction of an antisymmetric part of the interaction can reproduce the finite width of the torque curve. ${ }^{10}$

Starting from Eq. (3), we can easily understand that if the studied compound exhibits molecular hysteresis, the $\tau$ vs $B$ curve must reflect this behaviour. An example is reported in Fig. 17 for the Cp*ErCOT SMM already mentioned in Section 4.2. However, CTM suffers from a major limitation, that torque is identically zero when the field is zero, precluding the observation of classical hysteresis curves. On the other side, using CTM is possible to correctly measure butterfly-like hysteresis curves that are quite common in

\footnotetext{
10 Note that the symmetry lowering is necessary to reproduce the finite size of the step, because in $S_{6}$ symmetry the total effects of Dzyaloshinskii-Moriya vectors should vanish and a true $S=0$ ground state is expected.
} 

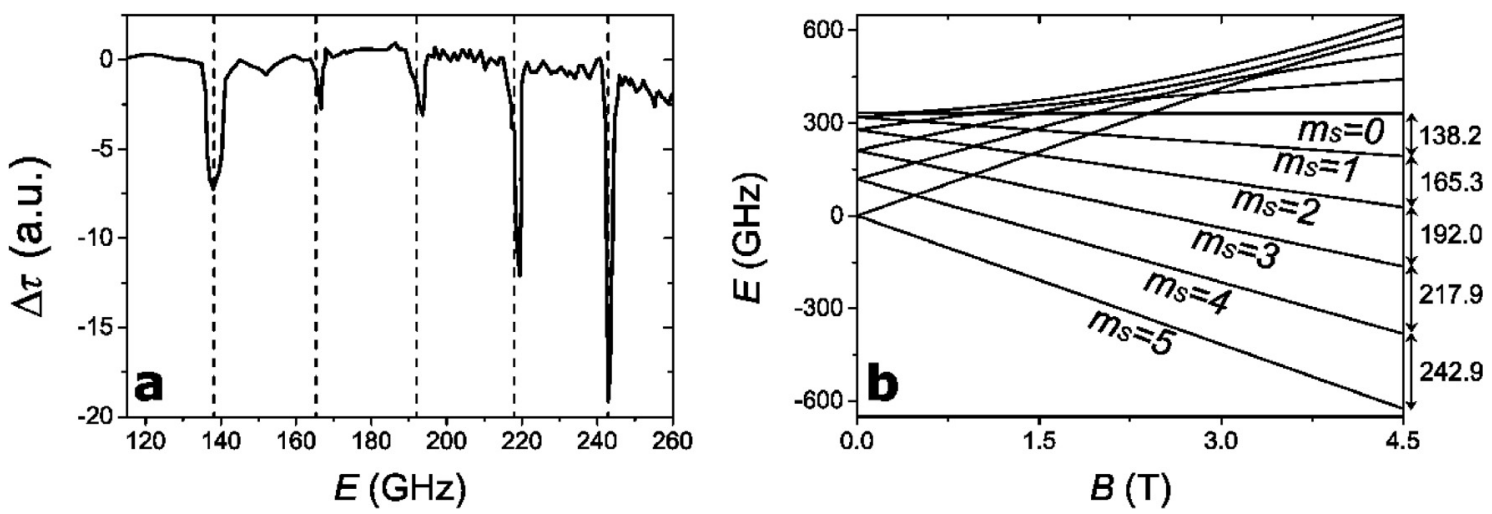

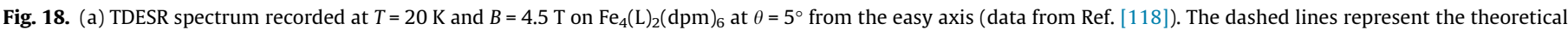
transition energies calculated from the Zeeman diagram (simulated using experimental conditions and parameters in Ref. [118]) reported in (b).

molecular compounds [91]. Although the exact shape of the curves is tricky to reproduce, a major information can be extracted: since the torque signal (initially positive), remains positive also when the field changes sign, it means that no remanence is present (magnetization flips its direction with the field), thus the hysteresis curve is a real butterfly-like hysteresis $[91,96]$.

\section{Torque detected electron spin resonance}

Several years ago, a new experimental set-up that merged the sensitivity and versatility of CTM with the spectroscopic accuracy of EPR was proposed [117]. This technique, known as Torque Detected Electron Spin Resonance (TDESR), requires an experimental set-up extremely similar to the one described in Section 3.2, but in addition irradiates the sample with light in the microwave energy range. The radiation can promote the transition between spin levels thus modifying the Boltzmann population. Since the magnetic torque at fixed temperature and magnetic field is clearly a sum of the torque produced by the single states weighted for the Boltzmann population, the detection of a different torque with and without irradiation delivers the same information provided by EPR. In Fig. 18a we reported the torque signal (more specifically, the torque difference with and without the microwave beam) versus the photons energy recorded by El Hallak et al. at $T=20 \mathrm{~K}$ and $B=4.5 \mathrm{~T}$ on a single crystal of $\mathrm{Fe}_{4}(L)_{2}(\mathrm{dpm})_{6}(\mathrm{Hdpm}=2,2,6,6$-tetra methyl-3,5-heptanedione, $\mathrm{H}_{3} L=(R, S)$-2-hydroxymethyl-2-(2-met hyl-butoxymethyl)propane-1,3-diol) [118]. The five peaks clearly visible in the graph, must be attributed to the transitions between the lowest spin states of the ground $S=5$ manifold. Assuming $b_{2}^{0}$ as the leading parameter in the CF Hamiltonian, the nonuniform spacing between the peaks (that lowers towards high frequencies) and the selection rule imposed by the microwave radiation perpendicular to both the magnetic field and the principal anisotropy axis $\left(\Delta m_{S}= \pm 1\right)$ already deliver the shape of magnetic anisotropy (easy axis). A simple simulation of a Zeeman diagram calculated using the parameters reported in the paper $\left(\theta=5^{\circ}\right.$ from the easy axis, $b_{2}^{0}=-0.148 \mathrm{~cm}^{-1}, b_{4}^{0}=1.1 \cdot 10^{-5} \mathrm{~cm}^{-1}$ and $\left.g=1.99\right)$ is pictured in Fig. 18b. The energies relative to the transition between states at $4.5 \mathrm{~T}$ are numerically reported on the right side of the Zeeman diagram and graphically plotted as dashed lines in Fig. 17a. The agreement between the experimental and calculated position of the resonances is extremely satisfactory. The TDESR experimental set-up was tested in several configurations to demonstrate the solidity of the method [117] and, more recently, on several coordination compounds with SMM properties $[118,119]$ confirming its broad applicability.

\section{Thin films}

The knowledge of the preferential order of molecules on a certain substrate is of paramount importance for many applications, however the information on the local order is normally very difficult to obtain. The main spectroscopic techniques that are used to characterize the average orientation of films are ellipsometry [120,121], EPR [122] and studies based on element/surface selectivity of synchrotron radiation [40]. On the other hand techniques based on electron beams such as transmission electron microscopy are very sensitive but need delicate sample preparation and are often performed in situ [123] due to the heavy impact of oxygen and moisture on the order of thin layers. Particularly challenging is the study of molecules that adopt a different orientation as a function of the film thickness $[124,125]$.

The high sensitivity to magnetic anisotropy typical of CTM allows the use of this technique to study not only single crystals but also films. Although magnetic films were studied since more than one decade [126-129], only recently the technique was extended to thin layers composed of anisotropic molecules [38].

In Fig. 19 we present the torque curves obtained at $T=2 \mathrm{~K}$ and $B=12 \mathrm{~T}$ on samples composed of ca. $100 \mathrm{~nm}$ of $\mathrm{TbPc}_{2}$ molecules evaporated on three substrates. The selected substrates were a metal (gold) an oxide (glass) and a highly conjugated organic molecule (perylene-3,4,9,10-tetracarboxylic dianhydride, PTCDA). The

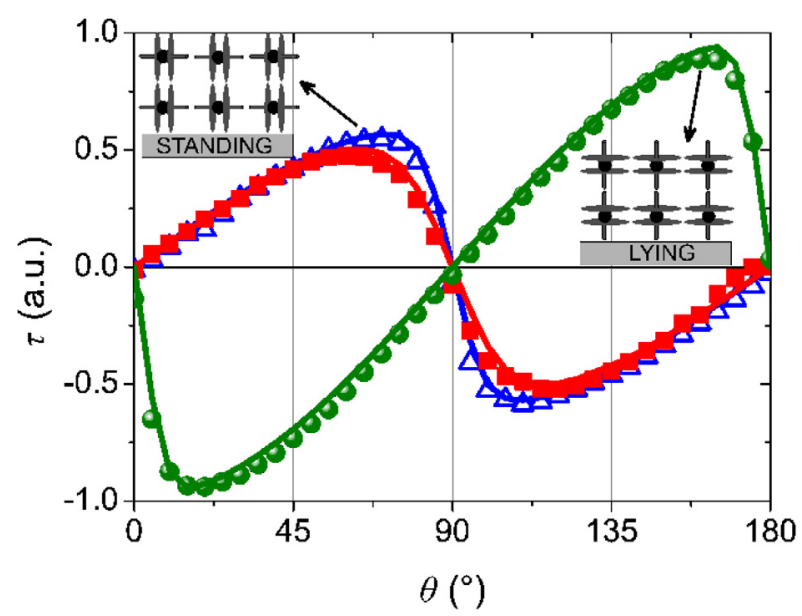

Fig. 19. Experimental data (symbols) and best fit (lines) for $100 \mathrm{~nm}$ of $\mathrm{TbPc}_{2}$ evaporated on PTCDA (green), gold (red) and glass (blue). Experimental conditions: $T=2 \mathrm{~K}, B=12 \mathrm{~T}$. 


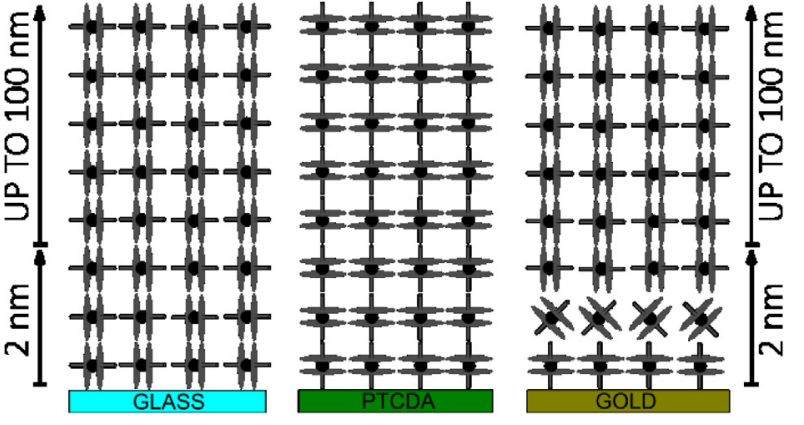

Fig. 20. Sketch of the molecular orientation of $\mathrm{TbPc}_{2}$ on glass, PTCDA and gold obtained interpreting CTM measurements.

different shape of the curves underlines an opposite molecular orientation of the film as a function of the selected substrate. The main orientation of the molecules on PTCDA is with the easy axis perpendicular to the surface (lying position) while the opposite holds for glass and gold (the easy axis parallel to the surface, standing position).

This result is however questionable, indeed the presence of small completely disordered regions can in principle be possible. To overcome this limitation, the authors propose an approach that exploits the unique sensitivity of CTM to magnetic anisotropy. The basic idea was to evaporate layers of $\mathrm{TbPc}_{2}$ buried in its diamagnetic analogue ( $\left.\mathrm{YPc}_{2}\right)$ to use magnetic anisotropy as a local probe to monitor the orientation of the film as a function of the distance from the surface. The use of the isostructural $\mathrm{YPc}_{2}$ assured an unperturbed growth of the film and at the same time a protection against air moisture.

The results, obtained assumed a Gaussian distribution centred on a most probable orientation [130], are depicted in Fig. 20. On chemically and thermally cleaned glass the $\pi-\pi$ stacking between Pc organic ligands is the main force that drives the packing [130], thus the molecules assumed a standing configuration (organic rings perpendicular to the surface). On the contrary, PTCDA evidenced a strong templating effect, forcing the molecules to assume a lying configuration (organic Pc rings parallel to the surface) even at a considerably high distance. This effect, clearly originated by the $\pi-\pi$ interaction between surface and ligands, was previously observed for similar systems [121,122], even if strongly dumped as a function of the layer thickness. However, the most intriguing result was found for the gold substrate. Although films of $5 \mathrm{~nm}$ or higher clearly showed a preferential standing orientation, for lower thicknesses no torque signal was detected. This lack of signal was attributed by the authors to a rapid reorientation from lying to standing, originating a disordered phase (already observed for CuPc molecules on gold [131]). To validate the results, some samples were analysed using synchrotron light, obtaining good agreement [38].

\section{Conclusion and future perspectives}

This review summarized the most relevant results obtained using CTM on coordination compounds in their solid state, enlightening that it represents a powerful resource to obtain precious experimental data and to validate theoretical models. Since CTM measurements are only sensitive to magnetic anisotropy (while the isotropic part of the signal is silent), the minimum size of the investigated crystals can be significantly smaller in comparison with other techniques such as SCM or PND. This effect is even more useful for crystals containing noncollinear anisotropies because it allows the disentanglement of the single contributions. Moreover, the quadratic field dependence of magnetic torque assures measurable signals even for moderate magnetic fields and allows to measure at much higher temperature compared to SCM. CTM was also used to extract energy levels splitting and wavefunction composition. This aspect can be strengthen combining CTM with microwave radiation.

Despite the many advantages just described, many suitable chemical systems and possible information that the technique can provide must still be explored. Concerning pure chemistry, a great opportunity is represented by Actinides containing complexes, that constitute an emergent field in molecular magnetism. A technique specifically accurate in determining magnetic anisotropy, such as CTM, could be extremely useful to provide information about the complex electronic structure of $5 f$ elements. Moreover, the study of thin films of anisotropic paramagnetic molecules is still at the beginning. Besides the huge number of molecular layers already reported in the literature as potential candidates in terms of spintronic applications but still not studied using CTM, a great opportunity is represented by mixed systems (e.g. alternating layers containing more than one anisotropic molecule).

Concerning the physical properties that can be extracted from CTM measurements, the author can foresee a great potential in the study of the single contributions to torque arising from different states inside a single manifold. This could be done either using chemically engineered systems (e.g. systems where symmetry imposes pure states) or performing a combined fit of torque and spectroscopic data.

\section{Acknowledgements}

The author wants to sincerely thank several people for the stimulating scientific discussion and useful suggestions: Prof. R. Sessoli, Prof. L. Sorace and Dr. E. Lucaccini (University of Florence), Prof. J. Van Slageren (University of Stuttgart), Prof. A. Cornia (University of Modena e Reggio Emilia), Prof. J. Bendix Dr. H. Weihe and Mr. M. A. Sørensen (University of Copenhagen). The help of Mr. Antonio Mazzoleni and Mr. L. Tesi as proof readers was kindly appreciated.

\section{Appendix A. Units and equations}

In this Appendix, the author discusses some mathematical definitions. Although most of the following paragraphs are not essential to perform a measurement, they intend to clarify some relevant topics concerning the formalism of equations.

\section{A.1. The units of torque}

In analogy with the classical definition of torque, magnetic torque is expressed in $\mathrm{N} \cdot \mathrm{m}$ (Newton times meter) in the International System (note the use of Newton first, to avoid the ambiguity with milliNewton, $\mathrm{mN}$ ). Dimensionally, $\mathrm{N} \cdot \mathrm{m}$ is equivalent to Joule, but the notation $\mathrm{N} \cdot \mathrm{m}$ is more appropriate for a quantity, such as torque, that is not a scalar (conversely to Energy). Indeed, torque contains also the angular dependence information, as explicitly visible in Eq. (3). In chemistry, it is common to scale $\tau$ per mole of substance, as the author did in this review. Moreover, the use of $\mathrm{Nm} / \mathrm{mol}$ is also handy because the torque exerted by a mole of an anisotropic coordination compound is often of the order of $10^{1}-10^{2} \mathrm{Nm} / \mathrm{mol}$. However, since most of the measurements performed in the literature were obtained from very small crystals of unknown mass, $\tau$ is often reported in arbitrary units.

\section{A.2. Magnetic field and magnetic induction}

From a mathematical point of view, the magnetic torque should have the same dimensions of an Energy $(J=N \cdot m)$. In the SI, this can 
be only obtained using the magnetic induction $\mathbf{B}$ (expressed in Tesla), because $\mathbf{M}$ is expressed in A. $\mathrm{m}^{2}=\mathrm{N} \cdot \mathrm{m} / \mathrm{T}$ (as reported in Eq. (3)). However, the physical quantity that the scientist has at disposal is the magnetic field generated by the instrument $\left(\mathbf{H}_{0}\right)$. For this reason, we need to derive an equation that relates the magnetic torque with the magnetic field.

In vacuum, the magnetic induction $\left(\mathbf{B}_{0}\right)$ is simply proportional to the magnetic field $\left(\mathbf{H}_{0}\right)$ :

$\mathbf{B}_{0}=\mu_{0} \mathbf{H}_{0}$

where $\mu_{0}$ is the vacuum permeability expressed in $\mathrm{Wb} /(\mathrm{A} \cdot \mathrm{m})$.

In materials, such as paramagnets, the relation between the magnetic induction $\mathbf{B}$ and the magnetic field $\mathbf{H}$ becomes more complicated due to the magnetization of the material (M, defined as the vector sum of all microscopic magnetic moments):

$\mathbf{B}=\mu_{0}(\mathbf{H}+\mathbf{M})$

Eq. (A.2) contains a quantity, $\mathbf{H}$, that is the "real" magnetic field inside the material considering both demagnetizing and shape effects, and is thus in principle slightly different from $\mathbf{H}_{0}$. However, for small samples (negligible shape influence), containing paramagnetic ions (diamagnetic contribution much smaller than the paramagnetic one), it is reasonable to assume $\mathbf{H}=\mathbf{H}_{0}$.

Substituting Eq. (A.2) into Eq. (3), and assuming $\mathbf{H}=\mathbf{H}_{0}$, we obtain:

$\tau=\mathbf{M} \times\left[\mu_{0}\left(\mathbf{H}_{0}+\mathbf{M}\right)\right]=\mu_{0} \mathbf{M} \times \mathbf{H}_{0}$

The vector product between the magnetization vector and itself is trivially zero, thus $\tau$ calculated using $\mathbf{B}$ or $\mathbf{H}_{0}$ are simply related by a constant $\left(\mu_{0}\right)$ in SI and are equal in CGS (remember that $\left.\mathbf{B}_{\mathbf{C G S}}=\mathbf{H}_{\mathbf{C G S}}+4 \pi \mathbf{M}_{\mathbf{C G S}}\right)$. Note that this expression was obtained assuming $\mathbf{H}=\mathbf{H}_{0}$ while no restrictions on $\mathbf{M}$ were imposed.

\section{Appendix B. Shape of torque curves}

\section{B.1. High field-limit}

The angular shape of the torque curves changes in the high field limit. Although there is no simple mathematical expression for this behaviour, it can be easily understood starting from Fig. A.1 where the angular dependence of $\mathbf{M}$ of a uniaxial system is reported.

The magnetization simulated at low field (blue curve) is cosinusoidal in shape, as expected when the field is rotated from the easy axis to the hard plane. For high fields, the situation changes dramatically. For angles close to the easy zeroes $\left(\theta=0^{\circ}\right.$ and $\theta=180^{\circ}$ ), the field causes saturation, thus the magnitude of $\mathbf{M}$ (already close to the highest possible) remains constant for a wide angular range, thus small variations of the free energy while scanning the angle are expected. Conversely, near the hard zero a small angular variation of $\mathbf{B}$ causes the appearance of a (small) component of $\mathbf{B}$ along $z$ that is capable to produce a huge $M_{z}$. Therefore, a massive change in the free energy occurs, and thus a rapid variation of $\tau$ (cf. Eq. (4)). For the same reason, the angle at which the magnetic torque is maximum also changes as a function of the field. To emphasize this difference, it is useful to plot the first derivative of $\tau$ with respect to the angle, as reported in Fig. A.2.

\section{B.2. Breaking field}

The position of the maximum in the $\tau$ vs $B$ plot at an angle close to the hard direction is influenced by several quantities such as ZFS parameter, value of the total spin and temperature. It can be intuitively understood that raising the spin and the ZFS have the effect to shift the breaking field towards high field values. This is the main reason why this kind of measurements are not suitable for lanthanides (with high values of the total angular momentum and large CF splitting). Also the temperature has a strong effect on the shape of the curve, as reported in Fig. A.3. High temperatures broaden the maximum (lowering at the same time the signal) and shift it at higher fields. Thus, the ideal Breaking Field measurement should be performed at low $T$ on anisotropic complexes containing light transition metals.

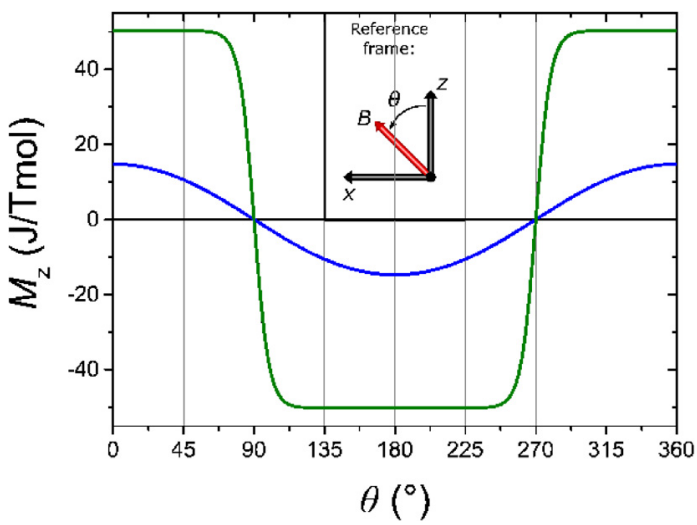

Fig. A1. Angular dependence of the projection of $\mathbf{M}$ along the $z$-axis at $T=2 \mathrm{~K}$ and $B=0.1 \mathrm{~T}$ (blue curve) ad $B=2 \mathrm{~T}$ (green curve) for a mole of $\mathrm{TbPc}_{2}$.

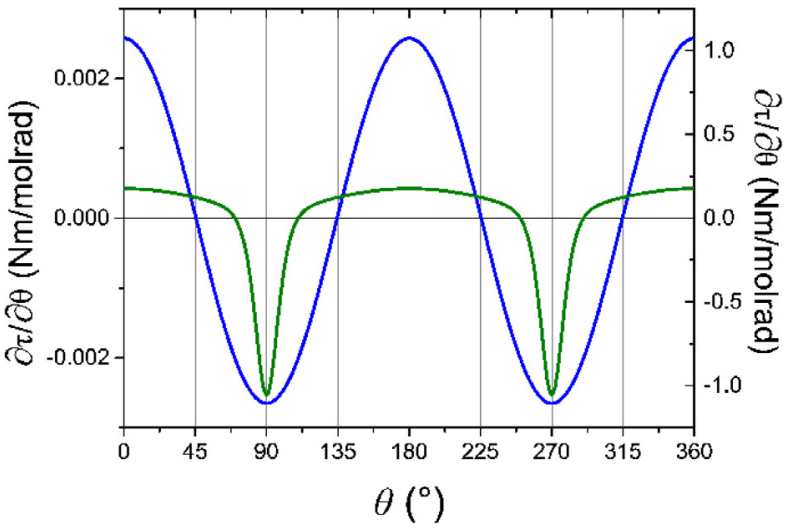

Fig. A2. First derivative of torque with respect to the angle for a mole of $\mathrm{TbPc}_{2}$ a $T=2 \mathrm{~K}$. Blue curve refers to $B=0.1 \mathrm{~T}$ (left scale) while green curve at $B=2 \mathrm{~T}$ (right scale).

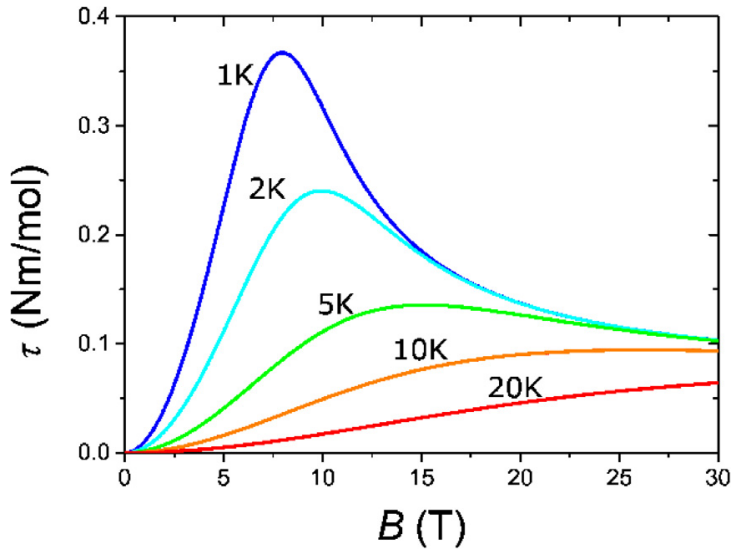

Fig. A3. Simulated temperature dependence of the torque signal for $S=2, \theta=89.9^{\circ}$ $\left(0.1^{\circ}\right.$ from the hard plane $)$ and $D=-5 \mathrm{~cm}^{-1}$. All the curves were divided by a factor $\sin \theta \cos \theta$ 

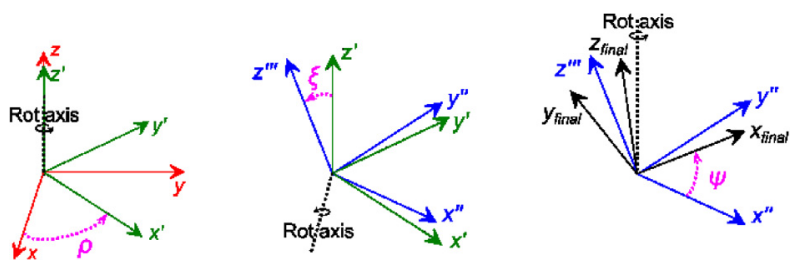

Fig. A4. Extrinsic rotation using the $x$-convention of the initial (red) reference frame using three Euler angles $(\rho, \xi, \psi)$ to obtain the final (black) reference frame.

\section{Appendix C. Reference frames and Euler angles}

The calculation and detection of magnetic torque is strictly related to the relative position of main magnetic axes and magnetic field. This requires a precise knowledge of the mutual position of several reference frames. In this Section, we will briefly explain the used reference frames and what is the connection between them.

The magnetic field direction is always known with great accuracy in the laboratory reference frame (XYZ). Normally, this reference frame is taken so that the magnetic field lies along $Z$ and the torque is measured along $Y$. This implies that the plane that is scanned during a rotation is always the $X Z$ plane.

The crystallographic reference frame $(a b c)$ can be trivially converted to the orthogonal reference frame $a b^{\prime} c^{*}$ following the standard crystallographic convention. Once the sample is indexed and placed on the cantilever, the relative position of $X Y Z$ and $a b^{\prime} c^{*}$ reference frames is known and fixed. It is thus possible to extract the director cosines of the vector $\mathbf{H}$ and of the vector $\boldsymbol{Y}$ in the crystallographic reference frame at the beginning of the rotation $\left(\theta=0^{\circ}\right)$.

Finally, the molecular reference frame ( $x y z$, defined as the reference frame where the susceptibility tensor is diagonal) should be connected to the crystallographic reference frame. The mutual orientation between these two reference frames is normally unknown and thus should be determined experimentally. The rotation matrix that connects two orthogonal reference frames can be always built up using three angles, called Euler angles (e.g. $\rho, \xi$, $\psi)$. They represent the angles of the three simple rotation matrices that must be subsequently applied to a reference frame to let it coincide with the new one. The elements of the $3 \times 3$ Euler matrix are clearly a function of these three angles and of the chosen convention for the rotation. Among the several possible conventions, we report in Fig. A.4 the three rotations that one must apply using the extrinsic $x$-convention.

The algebraic formula of the relative Euler matrix is as follows:

$$
\left(\begin{array}{ccc}
\cos \rho \cos \psi-\sin \rho \cos \xi \sin \psi & \cos \rho \sin \psi+\sin \rho \cos \xi \cos \psi & \sin \rho \cos \xi \\
-\cos \psi \sin \rho-\cos \rho \cos \xi \sin \psi & -\sin \psi \sin \rho+\cos \xi \cos \psi \cos \rho & \cos \rho \sin \xi \\
\sin \rho \sin \xi & -\sin \xi \cos \psi & \cos \xi
\end{array}\right)
$$

The order of the angles is $\rho, \zeta, \psi$ and the rotations are always anticlockwise. To find the director cosines of the main axes of the rotated reference frame in the initial reference frame is sufficient to invert the matrix (to save calculation time it is also possible to transpose the matrix, since for real orthonormal matrices the relation $\mathrm{A}^{\mathrm{T}}=\mathrm{A}^{-1}$ holds).

\section{References}

[1] L. Thomas, F. Lionti, R. Ballou, D. Gatteschi, R. Sessoli, B. Barbara, Nature 383 (1996) 145-147.

[2] M.N. Leuenberger, D. Loss, Nature 410 (2001) 789-793.

[3] J. Lehmann, A. Gaita-Arino, E. Coronado, D. Loss, Nat. Nanotechnol. 2 (2007) $312-317$.
[4] J. Lehmann, A. Gaita-Arino, E. Coronado, D. Loss, J. Mater. Chem. 19 (2009) $1672-1677$.

[5] M. Affronte, F. Trolani, A. Ghirri, S. Carretta, P. Santini, R. Schueeker, G. Timco, R.E.P. Winpenny, J. Magn. Magn. Mater. 310 (2007) E501-E502.

[6] A. Cornia, P. Seneor, Nat Mater 16 (2017) 505-506.

[7] E. Coronado, M. Yamashita, Dalton Trans. 45 (2016) 16553-16555.

[8] L. Bogani, W. Wernsdorfer, Nat. Mater. 7 (2008) 179-186.

[9] M.L. Perrin, E. Burzurí, H.S. van der Zant, Chem. Soc. Rev. 44 (2015) 902-919.

[10] D. Gatteschi, R. Sessoli, J. Villain, Molecular Nanomagnets, Oxford University Press, Oxford, UK, 2006.

[11] A. Abragam, B. Bleaney, Electron Paramagnetic Resonance of Transition Ions, Dover, New York, 1986.

[12] D.N. Woodruff, R.E. Winpenny, R.A. Layfield, Chem. Rev. 113 (2013) 51105148.

[13] R.A. Layfield, Organometallics 33 (2014) 1084-1099.

[14] R. Sessoli, A.K. Powell, Coord. Chem. Rev. 253 (2009) 2328-2341.

[15] H.L. Feltham, S. Brooker, Coord. Chem. Rev. 276 (2014) 1-33.

[16] G. Aromí, E.K. Brechin, Synthesis of 3d metallic single-molecule magnets, in: Single-molecule Magnets and Related Phenomena, Springer, 2006, pp. 1-67.

[17] G. Aromí, D. Aguila, P. Gamez, F. Luis, O. Roubeau, Chem. Soc. Rev. 41 (2012) 537-546.

[18] M. Nakano, H. Oshio, Chem. Soc. Rev. 40 (2011) 3239-3248.

[19] G. Cucinotta, M. Perfetti, J. Luzon, M. Etienne, P.E. Car, A. Caneschi, G. Calvez, K. Bernot, R. Sessoli, Angew. Chem. Int. Ed. 51 (2012) 1606-1610.

[20] M.E. Boulon, G. Cucinotta, J. Luzon, C. Degl'Innocenti, M. Perfetti, K. Bernot, G. Calvez, A. Caneschi, R. Sessoli, Angew. Chem. 125 (2013) 368-372.

[21] B.-W. Wang, Y. Meng, Y. Qiao, Y. Zhang, S. Jiang, Z. Meng, Z. Wang, S. Gao, Chem. Eur. J. 22 (2016) 4704-4708.

[22] D. Gatteschi, R. Sessoli, L. Sorace, Handbook on the Physics and Chemistry of Rare Earths, vol. 50, Elsevier, 2016, pp. 91-139, http://www.sciencedirect. com/science/article/pii/S0168127316300265.

[23] M.A. Sørensen, H. Weihe, M.G. Vinum, J.S. Mortensen, L.H. Doerrer, J. Bendix, Chem. Sci. 8 (2017) 3566-3575.

[24] S. Ghosh, S. Datta, L. Friend, S. Cardona-Serra, A. Gaita-Ariño, E. Coronado, S. Hill, Dalton Trans. 41 (2012) 13697-13704.

[25] S. Hill, S. Maccagnano, K. Park, R. Achey, J. North, N. Dalal, Phys. Rev. B 65 (2002) 224410.

[26] L. Sorace, M.-E. Boulon, P. Totaro, A. Cornia, J. Fernandes-Soares, R. Sessoli, Phys. Rev. B 88 (2013) 104407.

[27] J. Henderson, C. Koo, P. Feng, E. Del Barco, S. Hill, I. Tupitsyn, P. Stamp, D. Hendrickson, Phys. Rev. Lett. 103 (2009) 017202.

[28] S.D. Emerson, G.N. La Mar, Biochemistry 29 (1990) 1556-1566.

[29] I. Bertini, C. Luchinat, G. Parigi, Prog. Nucl. Magn. Reson. Spectrosc. 40 (2002) 249-273.

[30] I. Bertini, C. Luchinat, Coord. Chem. Rev. 150 (1996) 1-250.

[31] O.A. Blackburn, R.M. Edkins, S. Faulkner, A.M. Kenwright, D. Parker, N.J. Rogers, S. Shuvaev, Dalton Trans. 45 (2016) 6782-6800.

[32] Y. Takano, R. Tashita, M. Suzuki, S. Nagase, H. Imahori, T. Akasaka, J. Am. Chem. Soc. 138 (2016) 8000-8006.

[33] Y. Zhang, D. Krylov, M. Rosenkranz, S. Schiemenz, A. Popov, Chem. Sci. 6 (2015) 2328-2341.

[34] O.A. Blackburn, N.F. Chilton, K. Keller, C.E. Tait, W.K. Myers, E.J. McInnes, A.M. Kenwright, P.D. Beer, C.R. Timmel, S. Faulkner, Angew. Chem. 127 (2015) $10933-10936$.

[35] K. Ridier, A. Mondal, C. Boilleau, O. Cador, B. Gillon, G. Chaboussant, B. Le Guennic, K. Costuas, R. Lescouëzec, Angew. Chem. 128 (2016) 4031-4035.

[36] K. Ridier, B. Gillon, A. Gukasov, G. Chaboussant, A. Cousson, D. Luneau, A. Borta, J.F. Jacquot, R. Checa, Y. Chiba, Chem. Eur. J. 22 (2016) 724-735.

[37] A. Gukasov, P. Brown, J. Phys.: Condens. Matter 14 (2002) 8831.

[38] M. Perfetti, M. Serri, L. Poggini, M. Mannini, D. Rovai, P. Sainctavit, S. Heutz, R. Sessoli, Adv. Mater. (Weinheim, Ger.) 28 (2016) 6946-6951.

[39] E.M. Pineda, T. Komeda, K. Katoh, M. Yamashita, M. Ruben, Dalton Trans. 45 (2016) 18417-18433.

[40] L. Margheriti, M. Mannini, L. Sorace, L. Gorini, D. Gatteschi, A. Caneschi, D. Chiappe, R. Moroni, F. Buatier de Mongeot, A. Comia, F.M. Piras, A. Magnani, R. Sessoli, Small 5 (2009) 1460-1466.

[41] A. Cornia, M. Mannini, P. Sainctavit, R. Sessoli, Chem. Soc. Rev. 40 (2011) 3076-3091.

[42] S. Mitra, A. Gregson, W.E. Hatfield, R.R. Weller, Inorg. Chem. 22 (1983) 17291732.

[43] V.M. Nikitina, O.V. Nesterova, V.N. Kokozay, V.V. Dyakonenko, O.V. Shishkin, J. Jezierska, Inorg. Chem. Commun. 12 (2009) 101-104.

[44] R. González, R. Chiozzone, C. Kremer, F. Guerra, G. De Munno, F. Lloret, M. Julve, J. Faus, Inorg. Chem. 43 (2004) 3013-3019.

[45] L. Rigamonti, A. Cornia, A. Nava, M. Perfetti, M.-E. Boulon, A.-L. Barra, X Zhong, K. Park, R. Sessoli, Phys. Chem. Chem. Phys. 16 (2014) 17220-17230.

[46] A.L. Barra, A. Caneschi, A. Cornia, F.F. De Biani, D. Gatteschi, C. Sangregorio, R. Sessoli, L. Sorace, J. Am. Chem. Soc 121 (1999) 5302-5310.

[47] B.S. Tsukerblat, M.I. Belinski, V.E. Fainzil'berg, Sov. Sci. Rev. B. Chem. (Engl. Transl.) 9 (1987) 339.

[48] P. Erdös, J. Phys. Chem. Solids 27 (1966) 1705-1720.

[49] I. Dzyaloshinsky, J. Phys. Chem. Solids 4 (1958) 241-255.

[50] T. Moriya, Phys. Rev. 120 (1960) 91-98.

[51] T. Moriya, Phys. Rev. Lett. 4 (1960) 228-230.

[52] F. Cinti, M. Affronte, A.G.M. Jansen, Eur. Phys. J. B 30 (2002) 461-468. 
[53] A.L. Barra, A. Caneschi, A. Cornia, D. Gatteschi, L. Gorini, L.P. Heiniger, R. Sessoli, L. Sorace, J. Am. Chem. Soc. 129 (2007) 10754-10762.

[54] N.E. Chakov, S.C. Lee, A.G. Harter, P.L. Kuhns, A.P. Reyes, S.O. Hill, N.S. Dalal, W. Wernsdorfer, K.A. Abboud, G. Christou, J. Am. Chem. Soc. 128 (2006) 69756989.

[55] A. Cornia, R. Sessoli, L. Sorace, D. Gatteschi, A.L. Barra, C. Daiguebonne, Phys. Rev. Lett. 89 (2002) 257201.

[56] A. Caneschi, A. Cornia, A.C. Fabretti, S. Foner, D. Gatteschi, R. Grandi, L. Schenetti, Chem.-Eur. J. 2 (1996) 2329-2331.

[57] K.L. Taft, C.D. Delfs, G.C. Papaefthymiou, S. Foner, D. Gatteschi, S.J. Lippard, J. Am. Chem. Soc. 116 (1994) 823-832.

[58] O. Waldmann, L. Zhao, L.K. Thompson, Phys. Rev. Lett. 88 (2002) 066401.

[59] J. Schnack, Dalton Trans. 39 (2010) 4677-4686.

[60] H. Diep, Frustrated Spin Systems, World Scientific, 2013.

[61] C. Lacroix, P. Mendels, F. Mila, Introduction to Frustrated Magnetism: Materials, Experiments, Springer Science \& Business Media, Theory, 2011.

[62] G. Toulouse, in: M. Mezard, et al., (ed.), Spin Glass Theory and Beyond: an Introduction to the Replica Method and its Applications, World Scientific Press, 1987, pp. 99-103.

[63] J. Vannimenus, G. Toulouse, J. Phys. C: Solid State Phys. 10 (1977) L537-L542.

[64] S. Kirkpatrick, Phys. Rev. B 16 (1977) 4630-4641.

[65] M.L. Baker, G.A. Timco, S. Piligkos, J.S. Mathieson, H. Mutka, F. Tuna, P. Kozłowski, M. Antkowiak, T. Guidi, T. Gupta, PNAS 109 (2012) 19113-19118.

[66] V. Vieru, L. Ungur, L.F. Chibotaru, J. Phys. Chem. Lett. 4 (2013) 3565-3569.

[67] N. Ishikawa, M. Sugita, T. Okubo, N. Tanaka, T. Lino, Y. Kaizu, Inorg. Chem. 42 (2003) 2440-2446.

[68] G. Meyer, N.M. Amer, Appl. Phys. Lett. 53 (1988) 1045-1047.

[69] G. Meyer, N.M. Amer, Appl. Phys. Lett. 53 (1988) 2400.

[70] S. Alexander, L. Hellemans, O. Marti, J. Schneir, V. Elings, P.K. Hansma, M. Longmire, J. Gurley, J. Appl. Phys. 65 (1989) 164-167.

[71] S.S. Lee, R.M. White, Sens., Actuators A 52 (1996) 41-45.

[72] C. Lee, T. Itoh, T. Suga, Sens., Actuators A 72 (1999) 179-188.

[73] F.J. Giessibl, B. Trafas, Rev. Sci. Instrum. 65 (1994) 1923-1929.

[74] C. Rossel, P. Bauer, D. Zech, J. Hofer, M. Willemin, H. Keller, J. Appl. Phys. 79 (1996) 8166-8173.

[75] S. Kohout, J. Roos, H. Keller, Rev. Sci. Instrum. 78 (2007) 013903.

[76] D. Rugar, R. Budakian, H.J. Mamin, B.W. Chui, Nature 430 (2004) 329-332.

[77] C. Rossel, M. Willemin, A. Gasser, H. Bothuizen, G.I. Meijer, H. Keller, Rev. Sci. Instrum. 69 (1998) 3199-3203.

[78] R.D. Peters, Rev. Sci. Instrum. 60 (1989) 2789-2793.

[79] G. Ventura, M. Perfetti, Thermal Properties of Solids at Room and Cryogenic Temperatures, Springer, 2014.

[80] A. Cornia, A.G.M. Jansen, M. Affronte, Phys. Rev. B 60 (1999) 12177-12183.

[81] A. Cornia, M. Affronte, A. Gatteschi, A.G.M. Jansen, A. Caneschi, R. Sessoli, J. Magn. Magn. Mater. 226 (2001) 2012-2014.

[82] M. Perfetti, E. Lucaccini, L. Sorace, J.P. Costes, R. Sessoli, Inorg. Chem. 54 (2015) 3090-3092.

[83] F. El Hallak, P. Rosa, P. Vidal, I. Sheikin, M. Dressel, J. Van Slageren, Europhys. Lett. 95 (2011) 57002.

[84] M. Kanesato, T. Yokoyama, O. Itabashi, T.M. Suzuki, M. Shiro, Bull. Chem. Soc. Jpn. 69 (1996) 1297-1302.

[85] P.V. Bernhardt, B.M. Flanagan, M.J. Riley, Aust. J. Chem. 53 (2000) 229-231.

[86] E. Lucaccini, L. Sorace, M. Perfetti, J.-P. Costes, R. Sessoli, Chem. Commun. 50 (2014) 1648-1651.

[87] B.M. Flanagan, P.V. Bernhardt, E.R. Krausz, S.R. Lüthi, M.J. Riley, Inorg. Chem. 41 (2002) 5024-5033.

[88] B.M. Flanagan, P.V. Bernhardt, E.R. Krausz, S.R. Lüthi, M.J. Riley, Inorg. Chem. 40 (2001) 5401-5407.

[89] K.S. Pedersen, L. Ungur, M. Sigrist, A. Sundt, M. Schau-Magnussen, V. Vieru, H. Mutka, S. Rols, H. Weihe, O. Waldmann, Chem. Sci. 5 (2014) 1650-1660.

[90] J. Dreiser, C. Wäckerlin, M.E. Ali, C. Piamonteze, F. Donati, A. Singha, K.S. Pedersen, S. Rusponi, J. Bendix, P.M. Oppeneer, ACS Nano 8 (2014) 46624671.

[91] M. Perfetti, G. Cucinotta, M.E. Boulon, F. El Hallak, S. Gao, R. Sessoli, Chem. Eur. J. 20 (2014) 14051-14056.

[92] E. Lucaccini, M. Briganti, M. Perfetti, L. Vendier, J.P. Costes, F. Totti, R. Sessoli, L. Sorace, Chem. Eur. J. 22 (2016) 5552-5562.

[93] B. Cahier, M. Perfetti, G. Zakhia, D. Naoufal, F. El-Khatib, R. Guillot, E. Rivière, R. Sessoli, A.L. Barra, G. Nathalie, T. Mallah, Chem. Eur. J. 23 (2016) 3648-3657.

[94] B.W. Wang, S.D. Jiang, H.L. Sun, Z.M. Wang, S. Gao, J. Am. Chem. Soc. 133 (2011) 4730-4733.

[95] W.J. Evans, M.A. Johnston, R.D. Clark, J.W. Ziller, J. Chem. Soc., Dalton Trans. 10 (2000) 1609-1612.
[96] M.E. Boulon, G. Cucinotta, S.S. Liu, S.D. Jiang, L. Ungur, L.F. Chibotaru, S. Gao, R. Sessoli, Chem. Eur. J. 19 (2013) 13726-13731.

[97] M. Gysler, F. El Hallak, L. Ungur, R. Marx, M. Hakl, P. Neugebauer, Y. Rechkemmer, Y. Lan, I. Sheikin, M. Orlita, Chem. Sci. 7 (2016) 4347-4354.

[98] L. Rigamonti, C. Cotton, A. Nava, H. Lang, T. Rüffer, M. Perfetti, L. Sorace, A.L. Barra, Y. Lan, W. Wernsdorfer, Chem. Eur. J. 22 (2016) 13705-13714.

[99] A. Cornia, M. Affronte, A.G.M. Jansen, D. Gatteschi, A. Caneschi, R. Sessoli, Chem. Phys. Lett. 322 (2000) 477-482.

[100] I. Mihalcea, M. Perfetti, F. Pineider, L. Tesi, V. Mereacre, F. Wilhelm, A. Rogalev, C.E. Anson, A.K. Powell, R. Sessoli, Inorg. Chem. 55 (2016) 10068 10074.

[101] L. Gregoli, C. Danieli, A.L. Barra, P. Neugebauer, G. Pellegrino, G. Poneti, R. Sessoli, A. Cornia, Chem. Eur. J. 15 (2009) 6456-6467.

[102] S. Accorsi, A.L. Barra, A. Caneschi, G. Chastanet, A. Cornia, A.C. Fabretti, D. Gatteschi, C. Mortalo, E. Olivieri, F. Parenti, P. Rosa, R. Sessoli, L. Sorace, W. Wernsdorfer, L. Zobbi, J. Am. Chem. Soc. 128 (2006) 4742-4755.

[103] E. Tancini, M.J. Rodriguez-Douton, L. Sorace, A.L. Barra, R. Sessoli, A. Cornia, Chem. Eur. J. 16 (2010) 10482-10493.

[104] E.M. Kessler, S. Schmitt, C. van Wüllen, J. Chem. Phys. 139 (2013) 184110.

[105] I. Mihalcea, N. Zill, V. Mereacre, C.E. Anson, A.K. Powell, Cryst. Growth Des. 14 (2014) 4729-4734.

[106] S. Liu, L.-L. Li, H. Li, H.-L. Gao, J.-Z. Cui, P. Cheng, Dalton Trans. 44 (2015) 6169-6174.

[107] A. Cornia, D. Gatteschi, R. Sessoli, Coord. Chem. Rev. 219 (2001) 573-604.

[108] R. Sessoli, D. Gatteschi, A. Caneschi, M.A. Novak, Nature 365 (1993) 141-143.

[109] B.G. Wybourne, Spectroscopic Properties of Rare Earths, Interscience Publishers, New York, 1965.

[110] M. Kuz'min, J. Magn. Magn. Mater. 154 (1996) 333-338.

[111] N.W. Ashcroft, N.D. Mermin, Solid State Physics, Rinehart and Winston, New York, 1976.

[112] J.A.A.J. Perenboom, J.S. Brooks, S. Hill, T. Hathaway, N.S. Dalal, Phys. Rev. B: Condens. Matter 58 (1998) 330-338.

[113] J.A.A.J. Perenboom, J.S. Brooks, S. Hill, T. Hathaway, N.S. Dalal, Phys. B 246 (1998) 294-298.

[114] A. Cornia, M. Affronte, A.G.M. Jansen, G.L. Abbati, D. Gatteschi, Angew. Chem. Int. Ed. Engl. 38 (1999) 2264-2266

[115] J. Van Slageren, R. Sessoli, D. Gatteschi, A.A. Smith, M. Helliwell, R.E.P. Winpenny, A. Cornia, A.L. Barra, A.G.M. Jansen, E. Rentschler, G.A. Timco, Chem. Eur. J. 8 (2002) 277-285.

[116] M. Affronte, T. Guidi, R. Caciuffo, S. Carretta, G. Amoretti, J. Hinderer, I. Sheikin, A.G.M. Jansen, A.A. Smith, R.E.P. Winpenny, J. Van Slageren, D. Gatteschi, Phys. Rev. B 68 (2003) 104403.

[117] F. El Hallak, J. Van Slageren, M. Dressel, Rev. Sci. Instrum. 81 (2010) 095105

[118] F. El Hallak, P. Neugebauer, A.-L. Barra, J. Van Slageren, M. Dressel, A. Cornia, J. Magn. Reson. 223 (2012) 55-60.

[119] M. Dörfel, M. Kern, H. Bamberger, P. Neugebauer, K. Bader, R. Marx, A. Cornia, T. Mitra, A. Müller, M. Dressel, L. Bogani, J. van Slageren, Magnetochemistry 2 (2016) 25.

[120] P. Robaschik, P.F. Siles, D. Bülz, P. Richter, M. Monecke, M. Fronk, S. Klyatskaya, D. Grimm, O.G. Schmidt, M. Ruben, D.R.T. Zahn, G. Salvan, Beilstein J. Nanotechnol. 5 (2014) 2070-2078.

[121] P. Robaschik, M. Fronk, M. Toader, S. Klyatskaya, F. Ganss, P.F. Siles, O.G. Schmidt, M. Albrecht, M. Hietschold, M. Ruben, D.R.T. Zahn, G. Salvan, J Mater. Chem. C 3 (2015) 8039-8049.

[122] M. Warner, S. Mauthoor, S. Felton, W. Wu, J.A. Gardener, S. Din, D. Klose, G.W. Morley, A.M. Stoneham, A.J. Fisher, ACS Nano 6 (2012) 10808-10815.

[123] J.B. Gilchrist, T. Basey-Fisher, S.C.E. Chang, F. Scheltens, D.W. McComb, S. Heutz, Adv. Funct. Mater. 24 (2014) 6473-6483.

[124] D.G. de Oteyza, E. Barrena, J.O. Ossó, S. Sellner, H. Dosch, J. Am. Chem. Soc 128 (2006) 15052-15053.

[125] D.G. de Oteyza, E. Barrena, S. Sellner, J.O. Ossó, H. Dosch, J. Phys. Chem. B 110 (2006) 16618-16623.

[126] M. Lohndorf, J. Moreland, P. Kabos, N. Rizzo, J. Appl. Phys. 87 (2000) $5995-$ 5997.

[127] M.D. Chabot, J. Moreland, J. Appl. Phys. 93 (2003) 7897-7899.

[128] Y.-P. Hsieh, Y.-J. Li, Z.-H. Wei, M.-F. Lai, 2010 3rd International Nanoelectronics Conference (INEC), IEEE, 2010, pp. 529-530.

[129] J. Rigue, D. Chrischon, A. De Andrade, M. Carara, J. Magn. Magn. Mater. 324 (2012) 1561-1564

[130] I. Biswas, H. Peisert, M.B. Casu, B.E. Schuster, P. Nagel, M. Merzz, S. Schuppler T. Chasse, Phys. Status Solidi A 206 (2009) 2524-2528.

[131] I. Biswas, H. Peisert, M. Nagel, M.B. Casu, S. Schuppler, P. Nagel, E. Pellegrin, T. Chasse, J. Chem. Phys. 126 (2007) 174704. 